\title{
Berufliche Multilokalität und politische Partizipation: Warum Shuttles an ihrem Arbeitsort aktiv werden
}

\author{
Knut Petzold* \\ * Katholische Universität Eichstätt-Ingolstadt, Fachgebiet Soziologie, DE-85072 Eichstätt, knut. \\ petzold@ku.de.
}

\begin{abstract}
s
Berufliche Multilokalität und politische Partizipation: Warum Shuttles an ihrem Arbeitsort aktiv werden

Der Beitrag untersucht, ob und warum beruflich multilokalisierte Akteure (hier Shuttles) an ihrem Arbeitsort folgende politische Aktivitäten zeigen: Parteimitgliedschaft oder Bürgerinitiative, Teilnahme an politischen Veranstaltungen und lokale Spenden. Auf der Basis der Theorien Hirschmans, Olsons und Ajzens werden Hypothesen abgeleitet und im Rahmen einer multivariaten Vergleichsgruppenanalyse vorläufig geprüft. Es werden Daten einer Onlineerhebung unter den Nutzern einer Internetplattform für Mitfahrgelegenheiten und eines Businessnetzwerks genutzt. Es zeigt sich, dass auch Shuttles politisch aktiv sind, wobei sie insgesamt wenig zeitintensive Aktivitäten bevorzugen. Im Vergleich zu Locals scheint die politische Aktivität von Shuttles ausserdem stärker auf selektiven Anreizen zu beruhen.
\end{abstract}

Schlüsselwörter: Multilokalität, berufliche Mobilität, Shuttles, politisches Engagement, lokale Partizipation

Multilocalité professionnelle et participation politique: pourquoi les shuttles sont actifs sur leur lieu de travail

Cet article examine si et pourquoi les acteurs multilocalisés («shuttles») deviennent politiquement actifs sur leur lieu de travail en tant que membres locaux d'un parti, par la participation à une initiative citoyenne ou à des événements politiques ou par des dons. Sur la base des théories de Hirschmans, de Olson et de Ajzen, des hypothèses sont énoncées et testées dans le cadre d'une comparaison de groupe. Les données utilisées proviennent d'un sondage en ligne réalisé auprès des utilisateurs d'une plate-forme Internet pour covoiturage et d'un réseau d'entreprise. Les résultats montrent que, comparés aux acteurs monolocalisés («locals»), les shuttles aussi sont actifs politiquement, mais qu'ils privilégient les activités nécessitant un faible investissement de temps. De plus, leurs activités politiques sont davantage liées à des incitations sélectives.

Mots-clés: Multilocalité, mobilité professionelle, shuttles, engagement politique, participation locale

\section{Job-related Multi-locational Living and Political Participation: Why Shuttles Get Involved with Their Place of Work}

This paper examines whether and why professional commuters (shuttles) pursue political activities at their place of work such as: party membership, citizens' initiative, participation on political events and local donations. On the basis of the theories of Hirschman, Olson, and Ajzen hypotheses are derived and tested within a comparison group design using data from an online survey among users of an internet platform for ride sharing, as well as users of a business platform. The results show that shuttles are also politically active, but prefer less time-intensive activities. In addition, compared to locals the activities of shuttles are stronger related to selective incentives.

Keywords: Multilocation, job-related mobility, shuttles, political engagement, local participation 


\title{
Berufliche Multilokalität und politische Partizipation: Warum Shuttles an ihrem Arbeitsort aktiv werden ${ }^{1}$
}

\author{
Knut Petzold*
}

\section{$1 \quad$ Problemstellung}

Innerhalb der mobilen europäischen Bevölkerung ist das Pendeln über weite Distanzen, gekennzeichnet durch Übernachtungen ausserhalb des «eigenen» Wohnsitzes, eine präferierte Mobilitätsform (Meil 2008, 306; Dittrich-Wesbuer und Föbker 2013). Speziell in der Schweiz verfügen je nach Studie immerhin 8.5\% aller Haushalte (Bundesamt für Raumentwicklung 2009) bzw. ein Drittel der Schweizer Bevölkerung (Schad et al. 2015) über eine Zweitwohnung, von denen zwischen einem Fünftel und einem Viertel aus Gründen der Ausbildung oder des Berufs genutzt wird. Solche zyklischen Mobilitätsformen sind hauptsächlich das Resultat einer Strategie gegen Erwerbslosigkeit oder für beruflichen Aufstieg und werden durch verbesserte Verkehrsinfrastrukturen begünstigt. Die Pendelbereitschaft ist dabei allgemein unter Arbeitnehmern gestiegen und insbesondere unter Hochqualifizierten besonders stark ausgeprägt.

Diese komplexen zirkulären Formen moderner Mobilität werden in der Forschung seit einiger Zeit auch unter dem Begriff der Multilokalität diskutiert (z. B. Weiske et al. 2008; Weichhart 2009; Reuschke 2010; Petzold 2011; Petzold 2013a; Petzold 2013b; Hilti 2013; Rüger et al. 2014; Weiske et al. 2015). In einer Multilokalität verbinden die Akteure die Standortofferten verschiedener Wohnorte (vgl. Weichhart 2009). Multilokalität wird von Tagespendeln unterschieden, indem zu Ersterer mindestens eine zusätzliche Wohnung gehört, sodass der Begriff hier vor allem als residenzielle Multilokalität verstanden werden muss. Multilokalität ist aber kein fest definierter Begriff oder gar ein Forschungsparadigma, sondern umfasst je nach Perspektive sehr unterschiedliche Wohn- und Wanderungsphänomene (vgl. Hesse und Scheiner 2007). Was diese sehr unterschiedlichen Phänomene vor allem verbindet, ist eine aktive Mehrfachverortung der Akteure im Alltag.

Mobilität und Multilokalität wird in der sozialwissenschaftlichen Forschung vor allem aufgrund ihrer vermuteten Auswirkungen auf lokale Gemeinschaften,

Katholische Universität Eichstätt-Ingolstadt, Fachgebiet Soziologie, DE-85072 Eichstätt, knut. petzold@ku.de.

1 Ich danke den anonymen Gutachtern sowie den Herausgebern für ihre hilfreichen Anmerkungen zu einer früheren Version dieses Beitrags. 
etwa über lokale politische Partizipationsprozesse Aufmerksamkeit geschenkt, wobei regelmässig mindestens zwei konträre Positionen vertreten werden.

In einer eher pessimistischen Perspektive stehen Mobilität und Multilokalität der Entwicklung lokaler Orientierungen und Partizipation entgegen, da es zu «Entgrenzungen» und «Verflüssigungen» komme (Bonß und Kesselring 2002, 178). Häufige Umzüge und modernde Mobilität führten zu einem ziel- und bindungslosen Dahintreiben, das unter der Bezeichnung "Drift» Vertrauen und Verpflichtungen unterminiere (Sennett 1998, Kap. 1). Die aufgrund der Priorisierung beruflicher Entwicklung zunehmende Mobilität störe den Zusammenhang zwischen residenzieller Stabilität und lokalem Engagement (z. B. Putnam 1995; Putnam 2000). Dabei wird insbesondere die Gefahr sozialer Exklusion durch eine Verarmung an subjektiven Gestaltungsmöglichkeiten hervorgehoben, denn:

Am Herkunftsort ist man vielfach nicht mehr sozial, politisch und kulturell inkludiert, und am Zielort wird dies kaum zufriedenstellend erreicht. (Koch 2008, 222)

Allerdings wird in einer optimistischeren Perspektive auch immer wieder die massive Begünstigung lokaler Orientierungen gerade durch Mobilität hervorgehoben. Schon Sheller und Urry $(2006,210)$ betonen bei der Formulierung ihres "New Mobilities Paradigm", dass ein linearer Mobilitätsanstieg nur durch extensive Systeme der Immobilität flankiert werden kann. Empirische Studien zeigen, dass nicht obwohl, sondern weil längere und kürzere Abwesenheiten und kontrastierende Erfahrungen vorliegen, bereits vorhandene Beziehungen am Herkunftsort stärker ins Bewusstsein rücken (z. B. Case 1996). Gerade Mobilität erlaubt zudem die spontane Entwicklung neuer lokaler Bindungen über intensive Erfahrungen (Jorgensen und Stedman 2001). Hier ist Mobilität also Ursache der lokalen Orientierung.

The paradox that mobility might in fact be conductive to forming ties with a place is often overlooked. (van der Land 1998, 133)

Die Auswirkungen zunehmender räumlicher Mobilisierung auf lokale politische und soziale Partizipation und damit auf die Wohnorte generell werden demnach kontrovers diskutiert. An dieser Stelle möchte der Beitrag ansetzen, indem nach den Bedingungsgründen und der Häufigkeit politischer Partizipation bei multilokalen Akteuren gefragt wird. Um die situativen Bedingungen einer multilokalen Lebensführung möglichst konkret zu berücksichtigen, ist es notwendig, die Multilokalitätsform zu spezifizieren. So sollen sich die Aussagen in diesem Beitrag auf sogenannte Shuttles beziehen, die in der Literatur verhältnismässig gut beschrieben sind. Der Begriff geht auf Schneider et al. (2001) zurück und soll im Gegensatz zum Begriff des «Wochenpendlers» keine konkrete zeitliche Implikation enthalten. Eine operable Konkretisierung nimmt Reuschke (2010, 23-24) vor. Hiernach sind Shuttles Personen mit einer beruflich genutzten Zweitwohnung an einem dezidierten 
Zweitwohnsitz. ${ }^{2}$ Der Begriff des Shuttles beschreibt damit einen multilokalisierten Akteur, der zwischen einem Primär- und einem Arbeitsort pendelt. Die konkrete Forschungsfrage des Beitrags lautet entsprechend:

\section{Engagieren sich Shuttles politisch an ihrem Arbeitsort und wenn ja, unter welchen Bedingungen engagieren sie sich?}

Im Beitrag wird ein Vorschlag zur theoretischen Beantwortung der Forschungsfrage entwickelt und einer ersten, vorläufigen empirischen Prüfung unterzogen. Dabei wird in einem Kontrollgruppendesign eine multilokale Untersuchungsgruppe (Shuttles) mit einer unilokalen Kontrollgruppe (Locals) verglichen.

\section{Konzeptioneller Hintergrund und Hypothesen}

Die politikwissenschaftliche Forschung hat zahlreiche Definitionen politischer Partizipation vorgelegt, die meist zwei gemeinsame Aspekte beinhalten (vgl. Verba et al. 1995, 38; vgl. Gabriel und Völkl 2005, 528-529; Steinbrecher 2009, 27-29). Es handelt sich erstens um beobachtbare, zielgerichtete, freiwillige bürgerschaftliche Aktivitäten, die zweitens Entscheidungen im politischen System beeinflussen sollen. So definieren etwa Parry et. al. politische Partizipation als "action by citizens which is aimed at influencing decisions which are, in most cases, ultimately taken by public representatives and officials" (Parry et al. 1992, 16).

Darunter fallen entsprechend vielfältige Formen politischer Partizipation, die nach unterschiedlichen Dimensionen systematisiert werden können (vgl. Gabriel und Völkl 2005, 530-539; Bühlmann 2006, 38-39; Steinbrecher 2009, 44-50). Neben der rechtlichen Verfasstheit der Partizipation (z. B. Gabriel und Völkl 2005, 531) wird auch häufig ihre Unmittelbarkeit (z. B. für Wahlen: Verba et al. 1995) oder die Legalität (z. B. Opp 1992) bzw. Unkonventionalität (z. B. Gabriel 2013) zur Unterscheidung herangezogen. Parry et al. (1992) differenzieren politische Aktivitäten nach Stil, Qualität und Intensität.

Regelmässig werden die Wahlteilnahme, kommunikative Aktivitäten, Parteiaktivitäten, kollektive Aktivitäten, die förmliche Mitgliedschaft in Interessenverbänden, die Übernahme von Ehrenämtern, das Spenden von Geldbeträgen oder unkonventioneller Protest und Gewalt als Partizipationsformen genannt (Parry et al. 1992, 50, Gabriel und Völkl 2005, 532; Steinbrecher 2009, 47). Die folgende Auswahl an Partizipationsformen deckt hinsichtlich der Dimensionen eine gewisse Varianz ab und soll daher exemplarisch in dieser Untersuchung betrachtet werden. Als abhängige Variablen werden die Mitgliedschaft in einer lokalpolitisch aktiven Partei, die Beteiligung an einer lokalen Bürgerinitiative, das Spenden für lokale Organisationen

2 Multilokalitätsformen mit mehr als zwei Wohnstandorten werden in dieser Untersuchung demnach aus Gründen der Komplexität weder theoretisch noch empirisch berücksichtigt. 
und die Teilnahme an politischen Demonstrationen untersucht. ${ }^{3}$ Diese Formen politischen Engagements haben zudem einen direkten Einfluss auf die lokalen Strukturen und die lokale Gemeinschaft, sodass sie möglicherweise Hinweise auf den Wandel ganzer Orte und Regionen liefern.

In der Forschungsliteratur sind vielfältige individuelle und institutionelle Determinanten politischer Partizipation nachgewiesen worden, die sich insbesondere in sozioökonomische, kognitive, organisatorische Ressourcen sowie partizipationsrelevante Einstellungen und die Einbindung in soziale Netzwerke unterteilen lassen (vgl. Norris 2002; Gabriel und Völkl 2005; Bühlmann 2006; Steinbrecher 2009). Insbesondere in der politikwissenschaftlichen Partizipationsforschung hat sich das integrative Ressourcen-Sozialisations-Mobilisierungs-Modell von Verba et al. (1995) etabliert. Die Partizipation hängt hier von der individuellen Ressourcenausstattung des Akteurs, von den sozialisatorisch erworbenen partizipationsrelevanten Motiven sowie von seinem sozialen Umfeld ab, das ihn zur Partizipation anregt. Je stärker die drei Anreizfaktoren Ressourcen, Motive und soziale Netzwerke ausgeprägt sind, desto eher kommt es zu politischer Partizipation, wobei die relativen Gewichte empirisch variieren können. Zu den Motiven zählen insbesondere Werte, Normen und Interessen. Bei den Ressourcen wird durch die Autoren neben ökonomischen Mitteln sowie Wissen und Kompetenzen insbesondere die verfügbare Zeit als wesentlich hervorgehoben (Verba et al. 1995, 48).

Überträgt man diese Überlegungen auf die vorliegende Forschungsfrage, wird zunächst vor allem die knappe Ressourcenausstattung relevant. Denn eine multilokale Situation zeichnet sich gerade durch eine Knappheit an Zeit, an intensiven sozialen Kontakten sowie durch hohe kognitive Anforderungen und psychische Belastungen aus (z. B. Weiske et al. 2008, 290-295; Hilti 2013). Die allägliche Organisation mindestens zweier Wohnsitze, die Koordination von Familie bzw. Partnerschaft, die Planung der Fahrten und nicht zuletzt die tatsächliche temporäre Abwesenheit erfordern ein hohes Mass an Investition in Informationssuche und an zeitlicher Selbstdisziplin. Die «Transitionskosten» (Weichhart 2009, 9), bestehend aus Reise- und Kommunikationskosten, Kosten einer weiteren Wohnung usw., schlagen zusätzlich zu Buche. Die Kosten politischer Aktivitäten dürften durch Shuttles im Vergleich zu unilokalen Akteuren (Locals) daher grundsätzlich als höher wahrgenommen werden, was die Partizipationswahrscheinlichkeit senkt. Eine erste Hypothese lautet daher:

H1a: Bei multilokalen Akteuren kommt politisches Engagement insgesamt seltener vor als bei unilokalen Akteuren.

3 Diese Formen politischen Engagements sind an Mühler und Opp (2004; 2006) angelehnt und an der Operationalisierung in der Allgemeinen Bevölkerungsumfrage der Sozialwissenschaften (ALLBUS) des Leibniz-Instituts für Sozialwissenschaften (GESIS) orientiert (siehe auch: Gabriel und Völkl 2005). Auf die elektorale Partizipationsformen (Wahlteilnahme) ist hier bewusst verzichtet worden, da diese sich theoretisch und empirisch zu stark von den anderen Formen unterscheidet (vgl. Steinbrecher 2009, 51-54). 
Es ist aber auch denkbar, dass bei vorliegenden partizipationsrelevanten Motiven kostenintensive durch weniger kostenintenive Aktivitäten substituiert werden. So erfordert etwa die Mitgliedschaft in Parteien oder Bürgerinitiativen deutlich regelmässigere physische Präsenz als etwa die einmalige Teilnahme an politischen Demonstrationen. Demgegenüber kann sogar ganz ohne raumzeitlich spezifizierte eigene Anwesenheit und mit geringem Organisationsaufwand gespendet werden. Daher wird hinsichtlich des Aufwandes des Engagements angenommen:

H1b: Je niedriger der monetäre, zeitliche und organisatorische Aufwand des politischen Engagements ist, desto wahrscheinlicher engagiert sich ein multilokaler Akteur politisch am Arbeitsort.

Wird dem Ressourcen-Sozialisations-Mobilisierungs-Modell von Verba et al. (1995) gefolgt, sind politische Aktivitäten von Shuttles nur dann wahrscheinlich, wenn die durch die Multilokalität bedingte Zeit- und Ressourcenknappheit durch die beiden anderen Arten von Anreizfaktoren, also durch partizipationsrelevante Motive und die Einbindung in soziale Netzwerke, ausgeglichen wird. Unter multilokalen Bedingungen sollte politische Partizipation demnach im Vergleich zu unilokalen Bedingungen mit besonders hohen individuellen Anreizen einhergehen. Da das Modell von Verba et al. (1995) an dieser Stelle jedoch sehr unspezifisch ist, sollen die entsprechenden Mechanismen mithilfe dreier Theorien präziser expliziert werden.

\subsection{Politisches Engagement als kollektives Handeln}

Eine erste zweckmässige Anschlussmöglichkeit bietet die Theorie kollektiven Handelns (Olson 1965), die bereits häufig zur Erklärung politischer Partizipation, meist in der Form von Protest, angewendet wurde (z. B. Muller 1982; Opp 1986; Opp 1992; Opp und Finkel 2001). Politische Partizipation wird hier als gemeinsames Handeln von Personen zur Verbesserung des Angebots von Kollektivgütern aufgefasst. Ein Kollektivgut ist ein öffentliches Gut, von dessen Nutzung nach seiner Herstellung niemand sinnvoll ausgeschlossen werden kann (Olson 1965, 14). Nach der Theorie beteiligen sich Personen an der Herstellung eines Kollektivgutes, wenn sie eine Präferenz dafür besitzen, das heisst, ein Interesse daran entwickeln. Ein solches politisches Interesse gilt in der Partizipationsforschung als zentraler Prädiktor politischer Aktivität (z. B. Verba et al. 1995; Gabriel 2013).

Das Interesse allein ist aber nach der Theorie kollektiven Handelns noch nicht ausreichend. Zusätzlich müssen die Akteure glauben, dass das persönliche Engagement auch erfolgreich ist. Die Kernthese der Theorie ist nun, dass in grossen Gruppen dieser wahrgenommene Einfluss des Einzelnen so gering ist, dass es nicht zur Kollektivgutproduktion kommt. ${ }^{4}$ Um deren Herstellung zu gewährleisten, be-

4 Es zeigte sich in empirischen Studien aber, dass Personen ihren Einfluss nicht korrekt wahrnehmen, sondern überschätzen (z. B. Finkel und Muller 1998). Ein Direkteffekt des Interesses allein ist daher dennoch anzunehmen. 
darf es nach Olson zusätzlicher selektiver Anreize, also ökonomischer, sozialer oder psychischer Zusatznutzen oder Zusatzkosten.

In dieser Untersuchung sollen selektive Anreize zunächst über die Variable der lokalen Handlungsexklusivität betrachtet werden, die eine Kumulation der wahrgenommenen Einzigartigkeit eines Ortes durch den lokalen Akteur hinsichtlich der Realisierung seiner individuellen Präferenzen darstellt (Jorgensen und Stedman 2001, 233). Gerade für Shuttles kann der Arbeitsort schon allein aus Gründen der beruflichen Betätigung und des Einkommenserwerbs handlungsexklusiv werden. Es wird angenommen, dass mit einer steigenden wahrgenommenen Handlungsexklusivität aufgrund ihrer selektiven Anreizwirkung auch die Wahrscheinlichkeit politischer Partizipation steigt:

H2: (a) Je stärker das Interesse am Arbeitsort insgesamt ist und (b) je höher die wahrgenommene Handlungsexklusivität des Arbeitsortes ist, desto wahrscheinlicher engagiert sich ein multilokaler Akteur dort auch politisch.

\subsection{Abwendung, politisches Engagement und Identifikation}

Weitere selektive Anreize können aus der durch Hirschman (1970) formulierten Theorie zu "Exit, Voice, and Loyalty" abgeleitet werden, die bereits konkrete Elemente des Explanandums enthält. Die Theorie erklärt, wie Personen reagieren, wenn sich die Leistungen einer Organisation verschlechtern. Akteure können dann entweder mit Abwendung bzw. Abwanderung von dieser Organisation reagieren ("Exit") oder sie können sich um eine Verbesserung der Lage bemühen. Diese als "Voice" bezeichnete Alternative umfasst dabei von vorsichtiger Kritik bis zu gewaltsamen Protesten alle Versuche, eine Veränderung herbeizuführen. ${ }^{5}$ Akteure wählen umso eher die Voice-Alternative, wenn sie eine "Loyalty" gegenüber der Organisation empfinden. Diese Loyalty wird als "attachment to an organization" (Hirschman 1970, 77-78) beschrieben.

Die Theorie ist für Organisationen im weiteren Sinne, wie Firmen, Vereinigungen, Gewerkschaften, Parteien, Familien sowie Staaten entworfen worden (vgl. Dowding et al. 2000). Aufgrund des emotionalen Aspekts kann dabei Loyalty auch als Identifikation mit einer lokalen Gesellschaft verstanden werden (vgl. Mühler und Opp 2006, 293), die gerade bei Shuttles über Sozialisationsprozesse und eine positive Bewertung auch am Arbeitsort entstehen kann (Petzold 2013b). Da eine vorliegende lokale Identifikation mit erhöhten Kosten der Exit-Alternative (Scham, Gewissen), aber auch mit einem erhöhten Nutzen der Voice-Alternative (soziale Wertschätzung) einhergeht, ist die Wahrscheinlichkeit politischer Partizipation erhöht (vgl. Polletta und Jasper 2001; Mühler und Opp 2004; auch Gabriel 2013, 318): 6

5 Implizit wird auch die dritte Alternative der Inaktivität genannt.

6 Für berufliche Multilokalität ist anzunehmen, dass eine Abwanderung bzw. ein Abbruch der Multilokalität (Exit) deutlich kostspieliger ist, sodass es eher zur Aktivität (Voice) kommt. 
H3a: Je stärker die Identifikation eines multilokalen Akteurs mit seinem Arbeitsort ist, desto wahrscheinlicher engagiert er sich dort auch politisch.

Loyalty bzw. lokale Identifikation ist dann ein besonders starker Anreiz, wenn die Organisation bzw. lokale Gesellschaft in Schwierigkeiten ist, wenn der Akteur also mit den Bedingungen vor Ort unzufrieden ist. Das impliziert eine Interaktionsannahme zwischen der Identifikation und der Unzufriedenheit (vgl. De Weerd und Klandermans 1999, 1074; Mühler und Opp 2006, 244). Auch Unzufriedenheit wird in der Literatur immer wieder als Ursache politischer Partizipation diskutiert (z. B. Muller 1982; Opp 1992; Dalton 2004, 157-190). In dieser Studie wird dabei die Unzufriedenheit mit der lokalen Wohnsituation als zusätzlicher selektiver Anreiz betrachtet. Werden demnach die Minimalerwartungen an das Wohnen, wie Privatheit und Regeneration, am Arbeitsort nicht erfüllt, kann dies eine Bedingung für das Wirksamwerden von Identifikation darstellen:

H3b: Die positive Wirkung der Identifikation ist umso stärker, je grösser die Unzufriedenheit mit der Wohnsituation am Arbeitsort ist.

\subsection{Politisches Engagement als geplantes Verhalten}

Das Modell von Verba et al. (1995) betont ferner die Bedeutung von Normen und Werten sowie die Einbindung in soziale Gruppen für politische Partizipation. Tatsächlich zeigen zahlreiche Untersuchungen, dass die Anwesenheit signifikanter Anderer bzw. die Einbindung in soziale Netzwerke (z. B. Putnam 1995; Putnam 2000; Norris 2002; van Deth 2004; Gabriel 2013) für lokale politische Partizipation ebenso bedeutsam ist wie allgemeine Werte und normative Überzeugungen (z. B. Opp 1986; Pattie et al. 2004; Dalton 2008, 20-33; Gabriel 2013).

Dieser Einfluss kann mit der Theorie geplanten Verhaltens (Ajzen 1991) vertieft begründet werden. Nach dieser Theorie folgt Verhalten einer «Intention", die als das Ergebnis der Einstellung zu diesem Verhalten in Kombination mit der subjektiven Norm für oder wider dieses Verhalten und der wahrgenommenen Verhaltenskontrolle verstanden wird. Die subjektive Norm zu einem Verhalten ist dabei das Ergebnis des Zusammenspiels zwischen normativen Erwartungen signifikanter Anderer und der Motivation, sich diesen Erwartungen zu beugen. Moralische Vorstellungen, wie Normen, und soziale Erwartungshaltungen können demnach ebenfalls als selektive Anreize aufgefasst werden (vgl. Opp 1986; Finkel und Muller 1998; Opp und Finkel 2001, 76). Dabei wird angenommen, dass im Zeitverlauf nicht nur die soziale Einbettung als solche, sondern auch die Internalisierung lokaler Normen zunimmt. Entsprechend soll die lokale Wohndauer als Proxyvariable für die subjektive Norm herangezogen werden: 
H4: (a) Je länger ein multilokaler Akteur am Arbeitsort wohnt und (b) je mehr signifikante Andere am Arbeitsort anwesend sind, desto wahrscheinlicher engagiert er sich dort auch politisch.'

Zusätzlich zur Intention wird in der Theorie geplanten Verhaltens die wahrgenommene Verhaltenskontrolle als direkter Verhaltensprädiktor berücksichtigt. Da alle in einer Situation extern verfügbaren Ressourcen und Restriktionen das Handeln beeinflussen, kann ein und dieselbe Intention einmal zum Verhalten führen und ein andermal nicht. Für die Frage des politischen Engagements von Shuttles ist also ferner das Ausmass wichtig, mit dem ein multilokalisierter Akteur glaubt, überhaupt auf den Arbeitsort mit seinen politischen, sozialen und ökonomischen Strukturen Einfluss nehmen zu können.

H4c: Je stärker die wahrgenommene lokale Verhaltenskontrolle eines multilokalen Akteurs am Arbeitsort ist, desto wahrscheinlicher engagiert er sich dort auch politisch.

\subsection{Politisches Engagement am Arbeitsort im Vergleich zum Primärort}

Shuttles lassen sich aus beruflichen Gründen zusätzlich zum Primärort an einem Arbeitsort nieder. Der Primärort ist demnach länger bewohnt, weshalb dort insgesamt eine relativ stärkere soziale Integration und damit auch eine stärkere lokale Identifikation, ein grösseres Interesse und eine stärkere normative Erwartungshaltung signifikanter Anderer anzunehmen ist. Zusätzlich ist denkbar, dass bestimmte Handlungen am Primärort bereits vollzogen worden sind, was die normativen Erwartungen weiter verstärkt. Ferner dürfte der Primärort ganz besonders handlungsexklusiv sein, da der Akteur immer wieder dorthin zurückkehrt. Die vergleichsweise schwächere Involvierung der Shuttles am Arbeitsort dürfte auch mit einer schwächeren Überzeugung einhergehen, am Ort überhaupt etwas bewirken zu können. Das müsste insgesamt zu einer Asymmetrie des Engagements zwischen den Orten zugunsten des Primärortes führen.

\section{H5: Bei multilokalen Akteuren kommt politisches Engagement häufiger am Primärort und seltener am Arbeitsort vor.}

Aus der hier vertretenen Perspektive ist lokale politische Partizipation also zusammenfassend von der individuellen Ressourcenausstattung (v. a. Zeit) sowie von individuellen Motiven und der sozialen Einbettung abhängig. ${ }^{8}$ Die partizipationsrelevanten

$7 \quad$ Dabei handelt es sich um eine Unterstellung des Forschers, dass die Anwesenheit signifikanter Anderer normative Erwartungen zu politischen Aktivitäten impliziert. Es ist genau so gut denkbar, dass signifikante Andere eine Distanzierung von Politik erwarten. Hierauf wird in der Diskussion noch einmal eingegangen.

8 Lokale politische und gesellschaftliche Kontextbedingungen politischer Partizipation bleiben in dieser Studie aufgrund fehlender Aggregatdaten zu den Wohnorten der Shuttles und Locals unberücksichtigt. Darauf wird in der Diskussion noch einmal eingegangen. 
Motive werden hier als das Interesse am Ort, seine Handlungsexklusivität sowie als das Ausmass lokaler Identifikation und die Unzufriedenheit mit der Wohnsituation spezifiziert. Subjektive Normen werden über die Wohndauer approximiert. Die soziale Einbettung wird über die Anwesenheit signifikanter Anderer erfasst. Diese Merkmale werden als selektive Anreize zur politischen Partizipation aufgefasst. Ausgehend von der im Vergleich knappen Zeit- und Ressourcenausstattung bei Shuttles ist anzunehmen, dass diese selektiven Anreize besonders starke relative Gewichte entfalten müssen, damit politische Partizipation am Arbeitsort der mobilen Akteure beobachtet wird.

\section{Methodisches Design und Datengrundlage}

Um den Einfluss einer multilokalen Lebensführung auf lokale politische Aktivitäten zu untersuchen, wird eine multivariate Vergleichsgruppenanalyse mit einer multilokalen Untersuchungsgruppe und einer unilokalen Kontrollgruppe durchgeführt. Aufgrund mangelnder Messinstrumente der amtlichen Raumbeobachtung als auch aufgrund der Problematik, dass viele Multilokale Ihren Zweitwohnsitz nicht amtlich melden, finden sich praktisch kaum quantitative Angaben zu multilokalen Wohnformen (vgl. Sturm und Meyer 2009; Petzold 2011). Die wenigen Versuche der Quantifizierung beschränken sich zudem nur auf bestimmte Erhebungsregionen (vgl. Reuschke 2010; Dittrich-Wesbuer und Föbker 2013). Daraus ergeben sich zwei prinzipielle Probleme. Erstens ist eine Zufallsauswahl oder auch eine Quotierung entlang bestimmter Parameterverteilungen unmöglich. Zweitens liegt in der Rekrutierung eine grosse Herausforderung.

Aus diesen Gründen wurde hier das Design einer Online-Erhebung gewählt, das sich nicht auf ein konkretes Erhebungsgebiet bezieht (vgl. Nadler et al. 2015). Es ist gelungen, zur Rekrutierung der multilokalen Untersuchungsgruppe eine Kooperation mit dem Anbieter einer Internetplattform zur Organisation von Mitfahrgelegenheiten einzugehen und für einen Zeitraum von zwei Monaten im Sommer 2009 mobilisierte Personen zu rekrutieren. Dabei konnte davon ausgegangen werden, dass insbesondere Shuttles schon aufgrund der Ressourcenersparnis die Mitfahrgelegenheitsplattform intensiv frequentierten. Die Nutzer wurden aufgefordert, an der Befragung teilzunehmen, wenn sie selbst in irgendeiner Form an zwei oder mehr Wohnorten lebten. Die Rekrutierung der unilokalen Kontrollgruppe erfolgte innerhalb eines Monats im Jahr 2010 über ein grosses soziales Businessnetzwerk, da prinzipiell vergleichbare sozioökonomische Merkmale bei den Nutzern erwartet wurden. Es wurden Gesuche in 87 sogenannten «Regionalgruppen» gesetzt, in denen Personen derselben Region Kontakte knüpfen. Dabei wurde angenommen, dass in solchen Gruppen besonders viele Locals erreicht werden würden. Als Bedingung der Teilnahme wurde das Wohnen und Arbeiten an nur einem Ort formuliert. 
Unter den kompletten Fällen des kombinierten Datensatzes $(\mathrm{N}=1260)$ mussten im Nachhinein die Locals und die Shuttles identifiziert werden. Shuttles mussten berufstätig sein und angegeben haben, sich auch aus beruflichen Gründen multilokalisiert zu haben, um eine Abgrenzung von rein freizeitbedingten Multilokalitätsformen zu erreichen. ${ }^{9}$ Die Personen durften nicht seltener als mindestens einmal monatlich zwischen Primär- und Arbeitsort pendeln, um auch flexiblere Formen als das klassische Wochenendpendeln zu berücksichtigten. Um schliesslich das Kriterium des zusätzlich bezogenen Zweitwohnsitzes zu approximieren, ${ }^{10}$ musste die Wohndauer am Ausgangsort länger sein als am Arbeitsort. Um weiterhin Personen eindeutig als Locals zu identifizieren, mussten diese angegeben haben, dass sich an ihrem Wohnort auch ihr fester Arbeitsplatz befindet. Entlang dieser Kriterien konnten 313 Shuttles und 272 Locals identifiziert werden $(\mathrm{N}=585)$.

Tabelle 1 enthält die sozioökonomischen Merkmale der Shuttles und Locals sowie Angaben zu einer Vergleichsstichprobe (Reuschke 2010). Daraus geht eine spezifische, selektive Stichprobenzusammensetzung hervor. Es wird deutlich, dass es sich bei den rekrutierten Shuttles vorrangig um junge Erwachsene handelt, die sich in der beruflichen Etablierungsphase und vermutlich kurz vor der Familiengründung befinden. Diese Stichprobenzusammensetzung gründet möglicherweise in der spezifischen Nutzerklientel der genannten Onlineplattformen, was bei der Interpretation der folgenden Ergebnisse berücksichtigt werden sollte. Allerdings kommen die sozioökonomischen Parameter der Shuttles der Zusammensetzung der Vergleichsstichprobe sehr nahe, die Reuschke $(2010,60)$ in einem mehrstufigen Verfahren auf der Basis einer Zufallsauswahl aus den Melderegistern für die Städte München, Stuttgart, Düsseldorf und Berlin gezogen hat, was erlaubt, die Güte der Stichprobe etwas besser abzuschätzen. Die rekrutierte Kontrollgruppe der Locals ist den Shuttles in den Verteilungen des Alters, des Geschlechts, sowie der Bildung und beruflichen Stellung sehr ähnlich. Die Locals sind aber etwas häufiger verheiratet und haben öfter Kinder als die Shuttles, sodass nur ein annäherndes Gruppenmatching erreicht wurde.

Die gewählte Rekrutierungsstrategie hat möglicherweise Auswirkungen auf die Analyseergebnisse. Es ist prinzipiell denkbar, dass Mitfahrgelegenheiten als noch immer vergleichsweise unkonventionelles Verkehrsmittel systematisch durch Gruppen genutzt werden, die ein bestimmtes Niveau oder Muster politischen Engagements zeigen. So könnte diese Gruppe beispielsweise besonders sozial und solidarisch orientiert sein, sodass die Demonstrationsteilnahme überschätzt wird. Die

$9 \quad$ "Shuttles" sind definitionsgemäss primär beruflich multilokalisiert. Bei der analytischen Trennung nach beruflich und freizeitlich/familiär induzierten Multilokalisierungsmotiven ist jedoch die Komplementarität der Gründe zu berücksichtigen, da etwa Shuttles ja aus familiären Gründen auch stets zum Primärort zurückkehren (vgl. Hilti 2013).

10 Aus qualitativen Studien ist bekannt, dass die Frage nach Zweitwohnsitzen rechtliche Implikationen enthält, die oftmals nicht zutreffend sind (Hilti 2013). Daher wurde bei der Befragung auf sämtliche rechtlich konnotierten Begriffe verzichtet. 
Tabelle 1 Sozioökonomische Merkmale von Shuttles im Datensatz

\begin{tabular}{|c|c|c|c|}
\hline & Locals & Shuttles & $\begin{array}{r}\text { Reuschke } \\
(2010,81)\end{array}$ \\
\hline \multicolumn{4}{|l|}{ Alter (Jahre) } \\
\hline Wertebereich & $17-62$ & $19-64$ & $25-59$ \\
\hline Mittelwert (Standardabweichung) & $35.7(9.94)$ & $32.4(9.53)$ & $36.5(9.0)$ \\
\hline \multicolumn{4}{|l|}{ Geschlecht } \\
\hline Männlich & $46.7 \%$ & $53.0 \%$ & $60.6 \%$ \\
\hline Weiblich & $53.3 \%$ & $47.0 \%$ & $39.4 \%$ \\
\hline \multicolumn{4}{|l|}{ Familienstand } \\
\hline Ledig & $31.5 \%$ & $31.3 \%$ & $58.7 \%$ \\
\hline In fester Partnerschaft & $30.4 \%$ & $42.3 \%$ & k.A. \\
\hline Verheiratet & $31.8 \%$ & $20.3 \%$ & $36.4 \%$ \\
\hline $\begin{array}{l}\text { Geschieden/verwitwet } \\
\text { Getrennt lebend }\end{array}$ & $6.9 \%$ & $6.1 \%$ & $5.0 \%$ \\
\hline Kinder & $27.4 \%$ & $18.2 \%$ & $21.6 \%$ \\
\hline \multicolumn{4}{|l|}{ Höchster Berufsabschluss } \\
\hline Ohne Berufsausbildung & $7.3 \%$ & $4.0 \%$ & k.A. \\
\hline Facharbeiter & $13.3 \%$ & $14.8 \%$ & $15.2 \%$ \\
\hline Meister/Techniker & $8.5 \%$ & $7.7 \%$ & \\
\hline Fachschule & $8.8 \%$ & $4.0 \%$ & $\sum=8.8 \%$ \\
\hline Fachhochschule & $23.4 \%$ & $20.2 \%$ & \\
\hline Hochschule & $38.7 \%$ & $49.2 \%$ & $\Sigma=75.6 \%$ \\
\hline \multicolumn{4}{|l|}{ Berufliche Stellung } \\
\hline Selbstständig & $26.8 \%$ & $6.1 \%$ & Niedrig $=5.2 \%$ \\
\hline Beamter/äquiv. & $2.6 \%$ & $8.6 \%$ & Mittel = 24.5\% \\
\hline Arbeiter & $1.5 \%$ & $4.5 \%$ & Hoch $=70.4 \%$ \\
\hline Angestellter & $68.9 \%$ & $80.8 \%$ & \\
\hline \multicolumn{4}{|l|}{ Netto-Einkommen (Euro) } \\
\hline Wertebereich & $0-8000$ & $0-16000$ & $<2000$ \\
\hline Mittelwert & 1291.68 & 1829.95 & $58.9 \%$ \\
\hline (Standardabweichung) & (1 241.68) & $(1815.33)$ & \\
\hline Stichprobenumfang & 272 & 313 & 226 \\
\hline
\end{tabular}

Quelle: Eigene Berechnungen.

rein deskriptiven Analysen sind daher unter Vorbehalt zu interpretieren. Aufgrund des nicht perfekten Gruppenmatchings und der Selektivität der Stichprobe muss ausserdem bei der Prüfung der postulierten Zusammenhänge nach den Merkmalen 
des Alters, der Partnerschaft, der Kinder, des Bildungsgrades und des Einkommens statistisch kontrolliert werden, da hier weitere Einflüsse zu erwarten sind (vgl. Parry 1992; Verba et al. 1995; Keil 2012; Gabriel 2013). In den Modellen zu den Shuttles wird ausserdem auf die geplante Befristung der mobilen Lebensführung kontrolliert. Zwar sind auch die multivariaten Analysen aufgrund des Querschnittscharakters eingeschränkt, mithilfe der Kontrollvariablen sollte eine vorläufige Prüfung der postulierten Mechanismen zumindest im Sinne eines Falsifikationsversuchs aber gerechtfertigt sein.

\section{Ergebnisse}

\subsection{Häufigkeiten politischen Engagements bei Shuttles und Locals}

In Tabelle 2 sind die Häufigkeiten der politischen Aktivitäten der Shuttles an Primär- und Arbeitsort sowie die der Locals abgetragen. Die entsprechenden Fragen und Antwortkategorien sind Tabelle A1 im Anhang zu entnehmen.

Betrachtet man die Nennungen der Shuttles am Arbeitsort fällt auf, dass die Mitgliedschaft in einer Partei nur sehr selten erwogen und insgesamt kaum realisiert wird. Auch die Beteiligung an einer Bürgerinitiative tritt sehr selten auf, obwohl häufiger darüber nachgedacht wird. Dagegen haben etwa ebenso viele Personen bereits gespendet, wie diejenigen, die dies schon einmal in Erwägung gezogen haben. Mit fast einem Viertel haben sogar mehr Personen schon einmal an einer politischen Veranstaltung am Arbeitsort teilgenommen, als erwogen. Für Werte der politischen Aktivitäten am Primärort bestätigen sich diese Verhältnismässigkeiten ebenfalls. Parteimitgliedschaft und Bürgerinitiativbeteiligung kommen insgesamt gleich selten vor. Dagegen hat immerhin ein Drittel schon gespendet und sogar über die Hälfte schon an einer politischen Veranstaltung teilgenommen. In der Kontrollgruppe der Locals lässt sich das grundlegende Muster der Häufigkeiten politischen Engagements ebenfalls nachzeichnen.

Die deskriptiven Ergebnisse machen demnach deutlich, dass Hypothese 1a, nach der Shuttles insgesamt weniger politisch aktiv sein sollten als Locals, nicht zuzutreffen scheint. Zwar sind sämtliche Werte für die Shuttles am Arbeitsort erkennbar geringer, werden jedoch die Aktivitäten am Primärort ebenfalls berücksichtigt, lässt sich über beide Orte hinweg im Vergleich mit den Locals in etwa genauso häufig (Partei und Bürgerinitiative) und mitunter sogar häufiger (Spenden und Veranstaltungen) politisches Engagement unter den Shuttles ausmachen.

Gleichzeitig scheint Hypothese $1 b$ gestützt zu werden, in der aufgrund der vergleichsweise knappen Zeit- und Ressourcenausstattung von Shuttles ein selteneres Engagement bei höheren monetären, zeitlichen und organisatorischen Kosten angenommen worden ist. An beiden Orten sind die besonders zeit- und arbeitsintensiven Formen der Parteimitgliedschaft und Bürgerinitiativbeteiligung jeweils deutlich 
Tabelle 2

Häufigkeiten politischen Engagements bei Shuttles im Datensatz

\begin{tabular}{|c|c|c|c|c|}
\hline & \multirow[t]{2}{*}{ Locals (in \%) } & \multicolumn{2}{|c|}{ Shuttles (in \%) } & \multirow{2}{*}{$\begin{array}{l}\text { Korrelation } \\
\text { der lokalen } \\
\text { Aktivitäten } \\
\text { Shuttles }\left(\tau_{\mathrm{b}}\right)\end{array}$} \\
\hline & & Arbeitsort & Primärort & \\
\hline \multicolumn{5}{|c|}{ Mitglied in lokalpolitisch aktiver Partei } \\
\hline Kam nicht infrage & 65.9 & 90.6 & 70.3 & \multirow{4}{*}{$\begin{array}{c}0.423^{* *} \\
(\mathrm{~N}=308)\end{array}$} \\
\hline Überlegt, aber nicht gemacht & 21.8 & 7.1 & 20.3 & \\
\hline Einmal & 7.2 & 0.6 & 6.1 & \\
\hline Mehrfach & 5.2 & 1.6 & 3.2 & \\
\hline \multicolumn{5}{|l|}{ Bürgerinitiative } \\
\hline Kam nicht infrage & 69.0 & 86.9 & 72.1 & \\
\hline Überlegt, aber nicht gemacht & 17.0 & 10.3 & 18.6 & \multirow{3}{*}{$\begin{array}{c}0.333^{* *} \\
(\mathrm{~N}=311)\end{array}$} \\
\hline Einmal & 8.7 & 1.9 & 7.4 & \\
\hline Mehrfach & 5.2 & 1.0 & 1.9 & \\
\hline \multicolumn{5}{|l|}{ Politische Veranstaltungen } \\
\hline Kam nicht infrage & 28.6 & 61.8 & 34.2 & \multirow{4}{*}{$\begin{array}{c}0.342^{* *} \\
(\mathrm{~N}=306)\end{array}$} \\
\hline Überlegt, aber nicht gemacht & 15.5 & 15.2 & 10.0 & \\
\hline Einmal & 16.7 & 10.4 & 14.2 & \\
\hline Mehrfach & 39.3 & 12.6 & 41.6 & \\
\hline \multicolumn{5}{|l|}{ Spenden } \\
\hline Kam nicht infrage & 40.1 & 76.8 & 49.2 & \multirow{4}{*}{$\begin{array}{r}0.304^{* *} \\
(\mathrm{~N}=308)\end{array}$} \\
\hline Überlegt, aber nicht gemacht & 17.5 & 11.3 & 16.7 & \\
\hline Einmal & 22.2 & 6.8 & 13.2 & \\
\hline Mehrfach & 20.2 & 5.2 & 20.9 & \\
\hline
\end{tabular}

Anmerkungen: ** $p \leq 0.01 ; \tau$ : Rangkorrelationskoeffizient Kendalls Tau $b$.

Quelle: Eigene Berechnungen.

seltener als das wenig zeitaufwendige Spenden und die Teilnahme an politischen Veranstaltungen. Der Vergleich mit den Aktivitäten der Kontrollgruppe zeigt jedoch, dass auch bei den Locals zeit- und kostenintensives Engagement selten ist, sodass die Vermeidung nicht allein mit der multilokalen Lebensweise begründet werden kann. Es ist allerdings erkennbar, dass die kostenintensiven Formen am Arbeitsort der Shuttles insgesamt noch einmal deutlich seltener vorkommen als am Primärort oder in der lokalen Vergleichsgruppe. Am Arbeitsort der Shuttles stellt sich offensichtlich die Ressource Zeit doch als noch einmal besonders kostbar und damit restriktiv dar.

Die Ergebnisse sprechen ferner für das Zutreffen der Hypothese 5, nach der am Primärort stärkere Ausprägungen der Aktivitäten erwartet worden waren, da 
hierfür eine stärkere soziale Einbindung, stärkere lokale Orientierung sowie ein ausgeprägtes lokales Interesse angenommen wurden. Ein weiterer Grund liegt vermutlich schlicht in der längeren Wohndauer, die häufigere Aktivitäten wahrscheinlich macht. Die Zusammenhangsanalysen (Kendalls $\tau_{\mathrm{b}}$ ) weisen mit positiven Werten zwischen $\tau_{b}=0.304$ und $\tau_{b}=0.423$ zugleich aber darauf hin, dass Shuttles, die sich am Primärort politisch engagieren, dies grundsätzlich auch eher am Arbeitsort tun. Das zeigt, dass zwar eine Asymmetrie zwischen den Orten hinsichtlich politischen Engagements besteht, dass sich diese aber nur als schwaches Gefälle und nicht als Gegensatz darstellt.

Shuttles sind demnach tatsächlich auch am Arbeitsort politisch aktiv, allerdings seltener als am Primärort. Werden die Aktivitäten an beiden Orten zusammen betrachtet, ist insgesamt kein geringeres Engagement im Vergleich zur lokalen Kontrollgruppe zu erkennen. In gewissem Umfang zeichnen sich aber Substitutionsprozesse am Arbeitsort ab, da hier zeit- und ressourcenintensive Formen noch seltener berichtet werden als am Primärort oder in der lokalen Vergleichsgruppe.

\subsection{Bedingungen politischen Engagements am Arbeitsort}

Darüber hinaus wurden die Hypothesen zu den Bestimmungsgründen des politischen Engagements mit binären Logitmodellen geprüft (z. B. Menard 2010). ${ }^{11}$ Da die abhängigen Variablen des politischen Engagements vier Ausprägungen haben und jeweils linkssteil verteilt sind, wurden die Kategorien der mindestens einmaligen und der mehrfachen Aktivität zusammengefasst. Die zusammengefassten Variablen weisen demnach die Kategorie der Realisierung «habe ich einmal/mehrmals gemacht» mit der Referenzkategorie «kam für mich nicht infrage/habe ich überlegt, aber nicht gemacht» auf. ${ }^{12}$ Ferner sind die Aktivitäten der Mitgliedschaften in lokalpolitisch aktiven Parteien und in lokalen Bürgerinitiativen zusammengefasst worden, um die extreme Schiefe der Verteilungen etwas zu reduzieren. Die Zusammenfassung erscheint angesichts der systematischen Ähnlichkeiten gerechtfertigt. Allerdings sind die weiterhin schiefen Verteilungen der Aktivitäten eine ungünstige Voraussetzung für die multivariate Analyse, wodurch die Reichweite der Ergebnisse eingeschränkt bleibt.

Zunächst wurden vollstandardisierte Logitkoeffizienten $\left(\Omega^{s}\right)$ berechnet (Pampel 2000, 33), die die Interpretation der relativen Effektstärken innerhalb der einzelnen Modelle erlauben. Darüber hinaus wurde bei der Integration des multiplikativen Interaktionsterms über die Zentrierung der Variablen gleichzeitig Multikollinearität reduziert (Best und Wolf 2010, 841). Allerdings sind standardisierte Logitkoeffizienten intuitiv nur schwer verständlich und für Gruppenvergleiche nicht gut geeignet, sodass zusätzlich die durchschnittlichen marginalen Effekte (Average Marginal Effects,

11 Auch wenn es sich um ein strengeres statistisches Verfahren handelt, kann auf der Querschnittsdatengrundlage keine Kausalität an sich geprüft werden.

12 Zur Operationalisierung und den Skaleneigenschaften der unabhängigen Variablen siehe die Tabelle A1 im Anhang. Insbesondere die gebildeten Einstellungsskalen werden aufgrund der vielen, kurzen Intervalle und zur Reduktion der Modellkomplexität hier quasimetrisch behandelt. 
AMEs) berechnet worden sind (vgl. Mood 2010, 75). Diese werden aus Gründen der Interpretierbarkeit und Robustheit hier vorgezogen, obwohl mit ihnen wegen der Nichtlinearität ein Informationsverlust einhergeht (Best und Wolf 2010, 852). Aufgrund modellinhärenter Interaktionseffekte in logistischen Regressionen sind diese zudem weniger sensibel für explizit spezifizierte Interaktionseffekte, weshalb die vorhergesagten Wahrscheinlichkeiten visualisiert werden (vgl. Best und Wolf 2010, 840). Insgesamt werden also die grafisch abgebildeten durchschnittlichen marginalen Effekte der theoretisch relevanten Variablen sowie die konditionalen Effekte zur anschaulichen Interpretation herangezogen. Zur Dokumentation der Modellgüte wird das Pseudo-Mass Nagelkerke- ${ }^{2}$ eingesetzt (Backhaus et al. 2006, 449). Allerdings ist dieses Mass likelihood-basiert und bezieht sich daher nicht auf die erklärte Varianz der abhängigen Variablen, sodass ein Modellvergleich problematisch ist. Daher wurde zusätzlich das Akaike Informationskriterium (AIC) berechnet, das auch den Vergleich der Güte nicht genesteter Modelle erlaubt (Best und Wolf 2010, 844).

Es sind je drei Modelle zu jeder der drei politischen Partizipationsformen geschätzt worden (vgl. Onlineanhang, Tabelle A2) ${ }^{13}$; eines für die Shuttles, eines für die Locals und ein Gesamtmodell. Die Gesamtmodelle enthalten als Prädiktor zusätzlich die Gruppenvariable. Die Modelle der Shuttles enthalten neben den sozioökonomischen Kontrollvariablen eine Variable, die die geplante Befristung der multilokalen Situation wiedergibt. Die Gesamtmodelle dienen hier allein der Identifikation des kontrollierten Gruppeneffekts. Die Koeffizienten der Gruppenvariablen in den jeweiligen Gesamtmodellen bestätigen noch einmal, dass die Wahrscheinlichkeit für alle drei politischen Aktivitäten auch unter statistischer Kontrolle bei den Shuttles niedriger als bei den Locals ist (H1a). Dabei muss aber bedacht werden, dass die Modelle sich allein auf den Arbeitsort, nicht auf den Primärort der Shuttles beziehen.

Werden die gruppenspezifischen Modelle betrachtet, weisen die Werte des Pseudo- $\mathrm{R}^{2}$ und des AIC über die drei politischen Aktivitäten eine durchweg bessere Anpassung der Daten an die Modelle für die Shuttles im Vergleich zu den Locals auf. Das legt nahe, dass, obwohl Shuttles deutlich seltener am Arbeitsort politisch aktiv werden als Locals, die theoretisch abgeleiteten Prädiktoren für Shuttles etwas besser zuzutreffen scheinen. ${ }^{14}$ Die politischen Aktivitäten variieren demnach bei den Shuttles etwas stärker mit den formulierten Anreizen als bei den Locals. Gleichzeitig zeigen die Werte, dass Mitgliedschaft in einer Partei oder Bürgerinitiative für die

13 Der Onlineanhang kann auf der Internetseite des Autors unter www.knut-petzold.de/sjs_onlineanhang.pdf abgerufen werden.

14 Da das Sample selektiv ist und zusätzlich multiplikative Terme aufgenommen wurden, besteht die Gefahr von Multikollinearität und damit verzerrter Schätzer. Der Variance Inflation Factor (VIF) als Indikator für Multikollinearität weist jedoch für alle Modelle akzeptable Werte aus: $\mathrm{VIF}=1.87$ (Gesamt), VIF $=1.76$ (Locals) und VIF $=1.96$ (Shuttles). Damit wird insgesamt eher eine geringe Multilkollinearität nahegelegt. 
Abbildung 1a Bedingungen der Mitgliedschaft in Partei oder Bürgerinitiative bei Shuttles
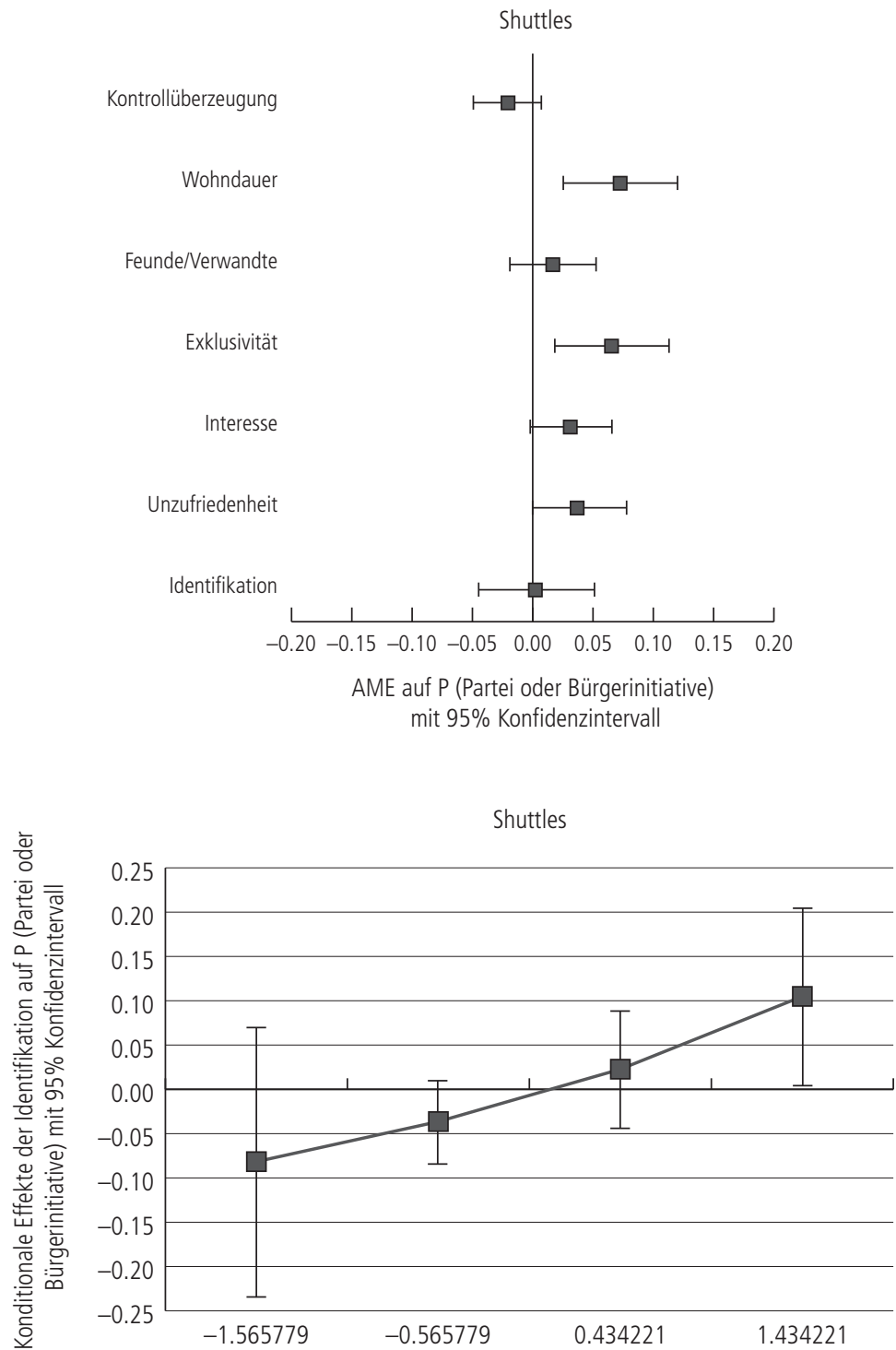

Z-Wert: Unzufriedenheit

Anmerkung: AME = Average Marginal Effects. Quelle: Eigene Berechnungen. 
Abbildung $1 \mathrm{~b} \quad$ Bedingungen der Mitgliedschaft in Partei oder Bürgerinitiative bei Locals
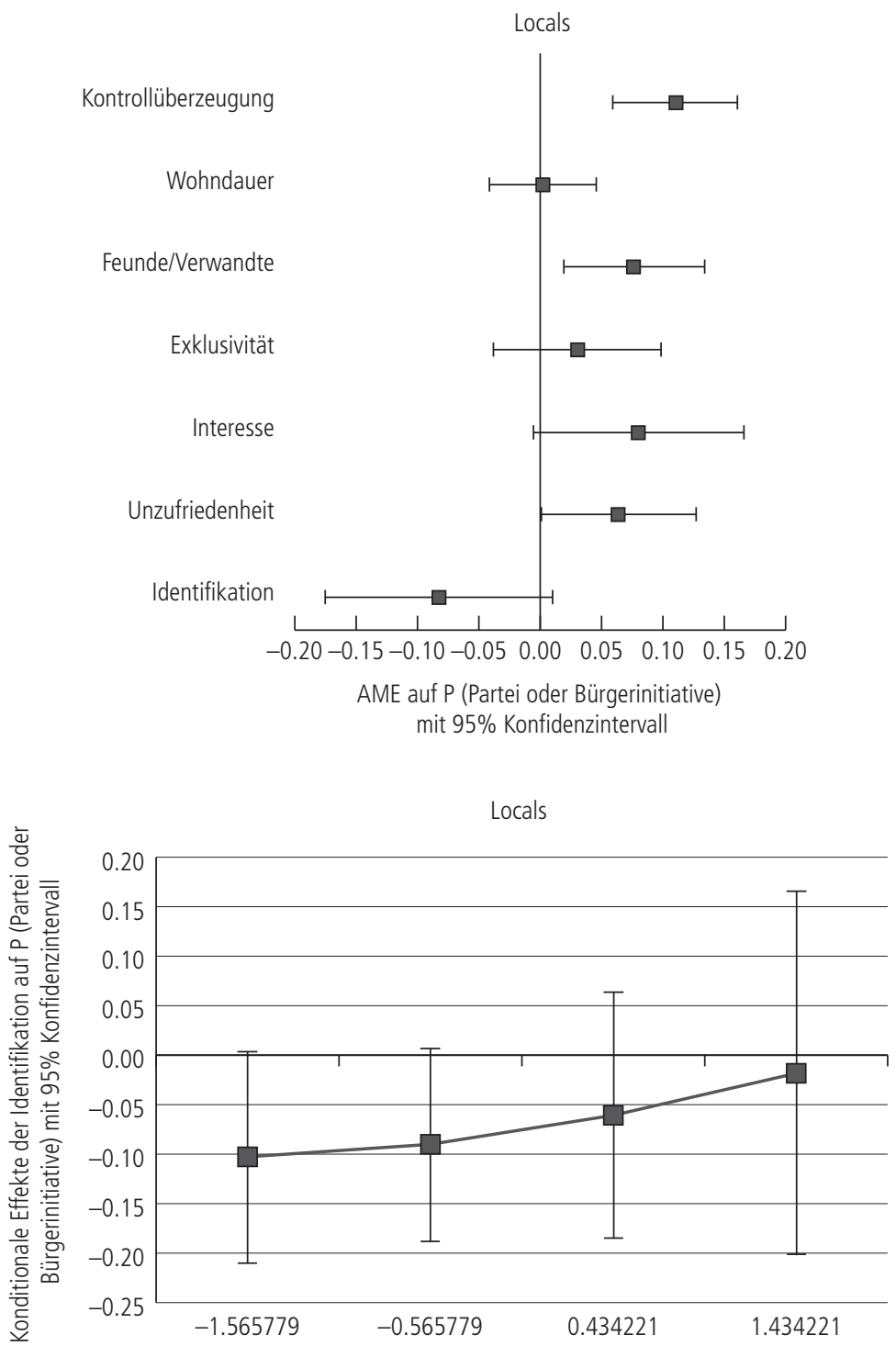

Z-Wert: Unzufriedenheit

Anmerkung: AME = Average Marginal Effects. Quelle: Eigene Berechnungen. 
Shuttles am Arbeitsort am besten durch das Modell erklärt werden kann und die Teilnahme an Demonstrationen am schlechtesten.

In Hypothese 2 wurde mit Verweis auf die Theorie kollektiven Handelns vermutet, dass das Interesse am Ort (H2a) und seine wahrgenommene Handlungsexklusivität (H2b) als selektive Anreize politische Partizipation fördern. Wie den Abbildungen 1 bis 3 zu entnehmen ist, werden beide Anreize in den Modellen zu den Shuttles jeweils nur einmal statistisch signifikant. So hängt das Interesse am Ort positiv mit der Teilnahme an politischen Veranstaltungen zusammen und die wahrgenommene Handlungsexklusivität geht mit einer Mitgliedschaft in Parteien und Bürgerinitiativen einher. Zwar ist auch im Modell zur Spendenwahrscheinlichkeit der Shuttles insbesondere der positive Effekt der Handlungsexklusivität annähernd statistisch bedeutsam, allerdings scheint sich die Gültigkeit der Überlegungen vorrangig für die anderen beiden Aktivitäten zu bestätigen. In den Modellen zur lokalen Kontrollgruppe wird das Interesse am Ort nur einmal für die Teilnahme an politischen Veranstaltungen statistisch bedeutsam.

In Hypothese 3 ist ferner angenommen worden, dass bei höherer Identifikation auch die Wahrscheinlichkeit für politisches Engagement höher ist ( $H 3 a$ ) und dass dieser Effekt durch eine Unzufriedenheit mit der lokalen Wohnsituation noch verstärkt wird (H3b). Die Schätzer zeigen, dass auf der Basis der vorliegenden Daten nur die Hypothese H3b und nur für die Mitgliedschaft in einer Partei oder Bürgerinitiative der Shuttles vorläufig bestätigt werden kann. Die lokale Identifikation allein zeigt in keinem der Modelle ein substanzielles Gewicht. Bei den Shuttles erweisen sich aber sowohl die Unzufriedenheit als auch die Interaktion mit der Identifikation als wichtige selektive Anreize für die Mitgliedschaft in einer Partei oder Bürgerinitiative am Arbeitsort. Dabei sind die marginalen Effekte der lokalen Identifikation mit jedem Schritt der Unzufriedenheit stärker, bis schliesslich bei höchster Unzufriedenheit dann auch der Effekt der lokalen Identifikation statistisch signifikant ist (Abbildung 1a). Der Interaktionseffekt ist zudem selbst auf dem 5\%-Niveau signifikant. Eine solche Interaktion zeigt sich auch im Modell zur Teilnahme an einer Demonstration bei den Shuttles, allerdings wird dieser Term nicht statistisch signifikant und auch die konditionalen Effekte erreichen keine statistisch bedeutsamen Werte. Im Modell zur Spendenwahrscheinlichkeit zeigt sich dagegen weder ein substanzieller Haupteffekt noch ein Interaktionseffekt (vgl. Abbildungen im Onlineanhang) ${ }^{15}$. Die Annahmen aus Hypothese 2 treffen darüber hinaus offensichtlich nicht für die lokale Kontrollgruppe zu, denn weder die lokale Identifikation noch die Interaktion mit der Unzufriedenheit (vgl. Abbildungen im Onlineanhang) erreichen in einem der Modelle bedeutsame Koeffizienten. Zudem weisen die konditionalen Effekte bei den Locals widersprüchliche Tendenzen auf, da die Interaktion einmal positiv, einmal negativ und einmal annähernd null ist. Damit erweisen sich insgesamt die

15 Der Onlineanhang kann auf der Internetseite des Autors unter www.knut-petzold.de/sjs_onlineanhang.pdf abgerufen werden. 


\section{Abbildung 2 Bedingungen der Teilnahme an politischen Veranstaltungen}

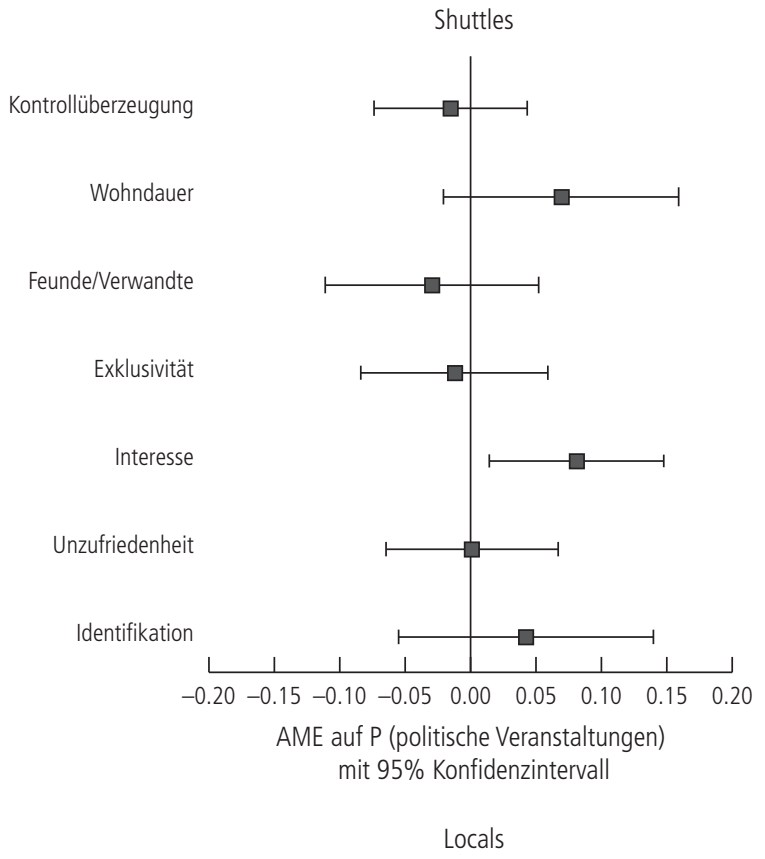

Kontrollüberzeugung

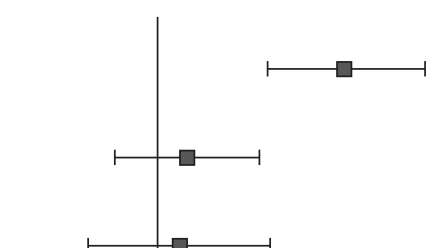

Feunde/Verwandte
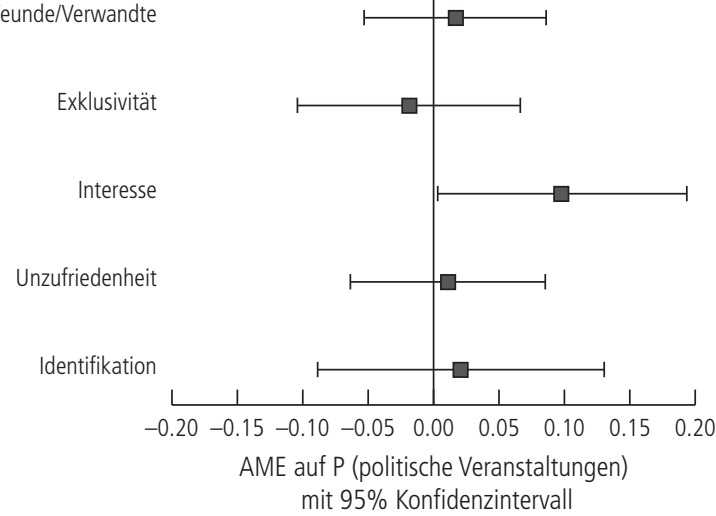

Anmerkung: AME = Average Marginal Effects. Quelle: Eigene Berechnungen. 


\section{Abbildung 3 Bedingungen der Spendenaktivität}
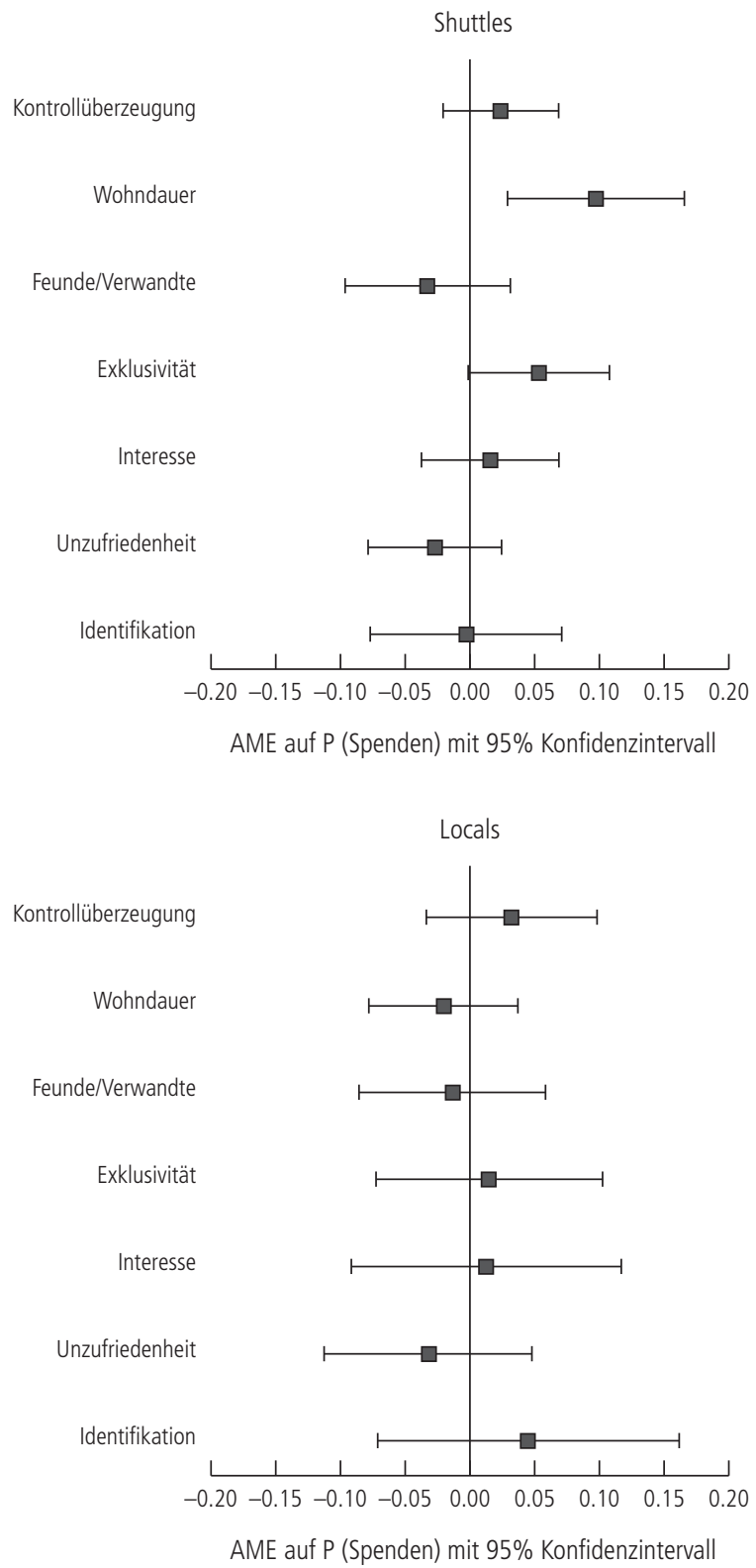

Anmerkung: AME =Average Marginal Effects. Quelle: Eigene Berechnungen . 
Annahmen aus der Theorie Hirschmans auf dieser Datengrundlage als besonders fruchtbar für die kostenaufwendige Mitgliedschaft in Parteien und Bürgerinitiativen am Arbeitsort der Shuttles.

Schliesslich wurde unter Bezugnahme auf die Theorie geplanten Verhaltens in Hypothese 4 postuliert, dass positive Effekte der Anwesenheit signifikanter Anderer (H4b), der lokalen Wohndauer als Proxyvariable für die lokale Norminternalisierung (H4a) und der wahrgenommenen lokalen Verhaltenskontrolle (H4c) zu erwarten sind. Diese Unterhypothesen können jedoch nicht mit den Daten belegt werden. Demgegenüber ist die lokale Wohndauer ein stabiler Prädiktor der Mitgliedschaft in einer Partei oder Bürgerinitiative und des Spendens bei Shuttles. Im Modell zur Demonstrationsteilnahme wird der Koeffizient der Wohndauer zwar nicht signifikant, ist aber ebenfalls positiv, sodass die Ergebnisse diese Unterhypothese dennoch weitestgehend zu stützen scheinen. Dagegen wird die Wohndauer in den Modellen der Locals überraschenderweise kein einziges Mal wirksam. Für die Kontrollgruppe ist dagegen in den Modellen zur Partei- und Initiativenmitgliedschaft und zur Veranstaltungsteilnahme die Überzeugung, am Ort auch etwas bewirken zu können, die stärkste Determinante. Im Modell zur Mitgliedschaft ist ausserdem die soziale Einbindung wichtig. Die Intention zur Mitgliedschaft und Veranstaltungsteilnahme basiert demnach bei Locals eher auf der lokalen wahrgenommenen Verhaltenskontrolle und bei Shuttles eher auf der vergleichsweise kurzen Wohndauer.

Bezüglich der Kontrollvariablen (siehe Onlineanhang, Tabelle A2) ${ }^{16}$ ist innerhalb der Gruppe der Shuttles bei Männern und Personen in einer Partnerschaft die Wahrscheinlichkeit einer Mitgliedschaft in einer Partei oder Bürgerinitiative grösser. Weiterhin wird das Spenden durch eine Partnerschaft etwas gehemmt. Ausserdem werden signifikante positive Bildungseffekte für die Spendenwahrscheinlichkeit ausgewiesen. Bei den Locals ist das Alter eine stabile positive Kovariate für alle drei Aktivitäten. Die Spendenwahrscheinlichkeit steigt hier zudem mit dem Einkommen. Insgesamt sichern die Kontrollvariablen mit grösserer Sicherheit die Gültigkeit der theoretisch relevanten Effekte.

\section{Diskussion und Fazit}

Ausgangspunkt dieser Untersuchung ist die Frage, inwiefern politische Aktivitäten am Arbeitsort unter den Bedingungen beruflicher Mobilisierung zu erwarten sind. Eine Annahme ist, dass eine mobile Lebensführung aufgrund einer besonderen Knappheit an Zeit bei einem gleichzeitig erhöhten organisatorischen Aufwand zu einer Erosion lokaler Aktivitäten führt. Allerdings kann Mobilität aufgrund kontrastierender Erfahrungen lokale Orientierungen und damit politische Aktivitäten

16 Der Onlineanhang kann auf der Internetseite des Autors unter www.knut-petzold.de/sjs_onlineanhang.pdf abgerufen werden. 
auch fördern, sodass der Forschungsstand sowohl theoretisch wie auch empirisch ergänzungsbedürftig ist. Auf der Basis des Ressourcen-Sozialisations-MobilisierungsModells von Verba et al. (1995), das mithilfe der Theorien zu Exit, Voice und Loyalty (Hirschman 1970), zu kollektivem Handeln (Olson 1965) und zu geplantem Verhalten (Ajzen 1991) spezifiziert worden ist, wurden Hypothesen zu den Determinanten politischer Partizipation bei Shuttles vorgeschlagen und mithilfe eines multivariaten Kontrollgruppendesigns auf der Basis einer Stichprobe aus Shuttles und Locals vorläufig geprüft.

Die Häufigkeiten der Aktivitäten am Arbeitsort (Tabelle 2) weisen nicht nur darauf hin, dass mobile Akteure im Vergleich zu lokalen Akteuren ihre politische Partizipation weiterhin aufrechterhalten, sondern legen auch nahe, dass kostenintensive und wenig kostenintensive Verhaltensweisen differenziert betrachtet werden sollten. Während vor allem zeit- und anwesenheitsintensive Beteiligungsformen (Mitgliedschaften) sowohl am Primär- als auch am Arbeitsort eher selten vorkommen, werden weniger aufwendige Formen (Spenden, Veranstaltungsteilnahme) deutlich häufiger berichtet. Dies trifft jedoch auch für die lokale Kontrollgruppe zu, sodass dieser Kosteneffekt nicht auf die mobile Lebensführung zurückgeführt werden kann. Allerdings werden die kostenintensiven Aktivitäten am Arbeitsort noch seltener realisiert als am Primärort. Das weist insgesamt darauf hin, dass die (zeitlichen) Knappheitsbedingungen bei beruflicher Mobilität politische Partizipation nicht grundsätzlich erodieren, sondern am Arbeitsort eher aufwendige Aktivitäten durch kostengünstigere Partizipationsformen substituiert werden.

Mit der Theorie Olsons ist angenommen worden, dass politische Partizipation von Shuttles vor allem von selektiven Anreizen abhängt. Neben der wahrgenommenen Handlungsexklusivität eines Ortes können weitere selektive Anreize mithilfe der Theorie Hirschmans als eine lokale Identifikation und die Unzufriedenheit mit der Wohnsituation spezifiziert werden. Die Theorie geplanten Verhaltens nach Ajzen erlaubt zudem, auch die soziale Einbettung und soziale Normen als selektive Anreize zu konzipieren.

Es zeigte sich in den Modellen zu den Shuttles, dass bei der besonders aufwendigen Mitgliedschaft in Parteien oder Bürgerinitiativen neben der Wohndauer als Proxyvariable der Norminternalisierung vor allem die wahrgenommene Handlungsexklusivität sowie die Unzufriedenheit mit der lokalen Situation in Interaktion mit der lokalen Identifikation zum Tragen kommen. In den Modellen zu den Locals wurde dagegen neben der lokalen Kontrollüberzeugung nur die soziale Einbettung bedeutsam. Während also gerade bei mobilen Akteuren eine ausgeprägte Unzufriedenheit vorliegen muss, damit die vergleichsweise junge lokale Identifikation für politische Partizipation wirksam wird, lassen sich die Effekte dieser selektiven Anreize bei den Locals nicht nachweisen. Im Modell zur Teilnahme an politischen Veranstaltungen wird bei den Shuttles insbesondere das vorliegende lokale Interesse bedeutsam. Bei den Locals ist dagegen wieder die lokale Kontrollüberzeugung wichtig. 
Schliesslich wurde im Modell zum Spenden bei den Shuttles ein signifikanter Effekt der Wohndauer und ein positiver, aber nicht sigifikanter Effekt der Handlungsexklusivität gefunden. Bei den Locals lässt sich dagegen gar kein interpretierbarer Effekt identifizieren.

Bei den Locals sind mit den Effekten der sozialen Einbettung und wahrgenommenen Verhaltenskontrolle vermutlich die Auswirkungen lang andauernder, intensiverer Sozialisation zu erkennen. Die Unzufriedenheit der Shuttles ist dagegen ebenso wenig von langwierigen Sozialisationsprozessen abhängig, wie das Interesse am Ort, die wahrgenommene Handlungsexklusivität oder eine lokale Identifikation. Eine erste Interpretation ist demnach, dass gerade aufwendige Formen politischer Partizipation bei beruflicher Mobilität selektive Anreize erfordern, die auch mehr oder weniger spontan entstehen können.

Die Analyse hat zudem gezeigt, dass die Modelle der Locals eine erkennbar schlechtere Anpassung an die Daten aufweisen und auch insgesamt weniger signifikante Koeffizienten enthalten als die Modelle der Shuttles. Das bedeutet aus statistischer Sicht, dass die eingesetzten Prädiktoren bei den Shuttles insgesamt stärker mit den berichteten politischen Aktivitäten variieren. Die formulierten selektiven Anreize können die Partizipation der Shuttles am Arbeitsort demnach besser erklären als die der Locals. Eine zweite Interpretation lautet daher, dass gerade unter den Bedingungen einer knappen Zeit- und Ressourcenausstattung, wie sie bei beruflicher Multilokalität häufig beobachtet wird, eindeutige zusätzliche selektive Anreize vorliegen müssen, damit eine politische Partizipation am Arbeitsort überhaupt wahrscheinlich wird. Im Vergleich zu Locals sind Shuttles am Arbeitsort zwar seltener politisch aktiv, wenn sie jedoch aktiv sind, dann stehen offenbar vergleichsweise klare selektive Anreize dahinter.

Werden die bedeutsamen Anreize in den jeweiligen Einzelmodellen betrachtet, könnte es zudem zweckmässig sein, zwischen starken und schwachen selektiven Anreizen zu unterscheiden. Während etwa das kognitive Interesse am Ort ein vergleichsweise schwacher Anreiz ist, wären eine emotionale Bindung an den Arbeitsort (Identifikation) und eine konkrete Unzufriedenheit starke Anreize. Aus dieser Sicht führen dann starke selektive Anreize auch eher zu kostenintensiven politischen Aktivitäten (z. B. Parteimitgliedschaft) und schwache Anreize zu weniger intensiven (z. B. Teilnahme an politischer Veranstaltung). So basiert etwa die Partei- oder Initiativenmitgliedschaft bei den Shuttles neben der Wohndauer vor allem auf starken Anreizen wie der Unzufriedenheit, in Interaktion mit der lokalen Identifikation, sowie auf der Handlungsexklusivität. Dagegen geht das Spenden bei den Shuttles beinahe allein auf die Wohndauer zurück. Dieses Muster ist bei den Locals nicht zu erkennen, da hier die lokale Verhaltenskontrolle am wichtigsten ist. Eine dritte Interpretation ist daher, dass insbesondere bei Shuttles stärkere Anreize auch mit kostenintensiven Aktivitäten einhergehen. 
Vor dem Hintergrund der Ergebnisse kann demnach insgesamt gemutmasst werden, dass eine Erosion lokaler politischer Partizipationsprozesse keinesfalls zwingend durch einen zunehmenden beruflich mobilisierten Teil der Bevölkerung zu erwarten ist. Die in dieser Untersuchung betrachteten Shuttles engagieren sich am Arbeitsort verhältnismässig gesehen in ähnlicher Weise wie an ihrem Primärort. Die befragten mobilen Akteure verfügen jedoch über eine charakteristische Ressourcenstruktur, in der nicht nur spezifische politische Aktivitäten bevorzugt werden, sondern diese auch in besonderem Masse auf zusätzlichen selektiven Anreizen basieren, die weniger zeitabhängig sind.

Gleichwohl unterliegt die Reichweite der Interpretation methodischen Einschränkungen, die sich vorrangig aus der Datengrundlage und der Operationalisierung der Konzepte ergeben.

So ist beispielsweise keine Einstellung in die Modelle eingegangen, die sich konkret auf das politische Engagement bezog. Die Präferenz zur Kollektivgutproduktion ist hier etwa über ein allgemeines Interesse an den Geschehnissen am Ort berücksichtigt worden, nicht aber über ein Interesse an politischen Aktivitäten selbst. Die verwendeten Operationalisierungen der selektiven Anreize sind demnach möglicherweise unzureichend. So ist denkbar, dass selektive Anreize zu politischem Engagement auch in einem Demokratieinteresse, in einem Interesse an Menschenrechten, in Religionszugehörigkeiten usw. liegen können. Auch die Operationalisierung der subjektiven Norm zu politischer Partizipation ist problematisch, denn sowohl die Anzahl der Freunde und Verwandten am Ort als auch die lokale Wohndauer sind nur sehr allgemeine und damit grobe Indikatoren für Norminternalisierungen und normative Erwartungen.

Zudem ist die Operationalisierung der politischen Partizipation selbst möglicherweise verkürzt. Mit der formellen Mitgliedschaft in einer Partei oder Bürgerinitiative sind beispielsweise noch keine spezifischen Teilnahmepflichten verbunden. Die Teilnahme an politischen Veranstaltungen kann auch rein informativen Charakter haben und damit eher politisches Interesse als aktive politische Teilnahme indizieren. Ferner können Geldspenden auch beliebig geringfügig und damit äusserst unverbindlich sein. Schliesslich sind die herangezogenen Formen politischer Partizipation ggf. nicht ganz trennscharf im Hinblick auf ihren lokalen oder überlokalen Bezug, da eine lokale Veranstaltung etwa auch ein globales Problem thematisieren könnte. Auch die Rolle sozialer Medien wurde hier nicht thematisiert, die möglicherweise insbesondere bei beruflicher Mobilität für politische Partizipationsprozesse eine herausragende Stellung einnehmen.

Die Modellanpassungen weisen ausserdem auf weitere Einflussfaktoren hin, die hier unbeachtet blieben. Einerseits könnten Persönlichkeitsfaktoren (z. B. die Big Five: Neurotizismus, Extraversion, Offenheit für Erfahrung, Gewissenhaftigkeit und Verträglichkeit) eine Rolle spielen. Andererseits sind generelle ideologische Überzeugungen (z. B. Einstellung zur Demokratie) nicht erfasst worden. Die be- 
merkenswerten positiven Korrelationen der politischen Aktivitäten zwischen dem Primär- und dem Arbeitsort liefern für solche Faktoren erste Indizien.

Ferner blieben in dieser Studie mögliche politische, soziale und ökonomische Kontexteffekte unberücksichtigt, da geeignete Aggregatdaten aufgrund der gewählten Rekrutierungsstrategie fehlten. So ist zum Beispiel denkbar, dass die Transformation der selektiven Anreize in tatsächliche politische Aktivitäten von spezifischen lokalen Voraussetzungen, wie etwa dem Vorhandensein örtlicher politischer Gruppen, sozialer Netzwerke oder Entscheidungsinstanzen abhängt.

Schliesslich wurden sozioökonomische Bedingungen der Individuen hier nur als Kovariaten zur statistischen Kontrolle der theoretisch interessierenden Anreizeffekte behandelt. Es wäre lohnenswert, diese Effekte stärker im Anschluss an die vorliegende Forschung zu systematisieren (z. B. Parry et al. 1992; Verba et al. 1995; Keil 2012; Gabriel 2013).

Die genutzten Daten stellen demnach nur eine erste Auswertungsgrundlage dar und bieten allenfalls einen explorativen Einstieg in die systematisch-analytische Auseinandersetzung mit dem Gegenstand politischer Partizipation bei beruflicher Mobilität. Eine Replikation mit differenzierteren Messinstrumenten auf einer breiteren Datenbasis ist demnach sehr vielversprechend. Dabei sollten nicht nur andere Operationalisierungen der sozialen Anreize und der politischen Partizipationsformen zur Anwendung kommen, auch die Berücksichtigung von Kontexteffekten und der systematische Vergleich lokaler und überlokaler politischer Partizipation wären wünschenswert. Darüber hinaus bleibt zu hoffen, dass insbesondere für die Schweiz nicht nur eine bevölkerungsrepräsentative Erfassung multilokaler Lebensformen entwickelt wird, sondern auch eine empirisch fundierte Beschreibung des Wandels von Ortschaften und Regionen durch zunehmende berufliche Mobilität. Denn die Frage der Auswirkungen zunehmender Mobilität auf lokale Strukturen muss so lang spekulativ bleiben, wie belastbare empirische Strukturdaten zum mobilitätsbedingten Wandel von Orten und Regionen fehlen.

\section{$6 \quad$ Literaturverzeichnis}

Ajzen, Icek. 1991. The theory of planned behavior. Organizational Behavior and Human Decision Processes 50(2): 179-211.

Backhaus, Klaus, Bernd Erichson, Wulff Plinke und Rolf Weiber. 2006. Multivariate Analysemethoden. Eine anwendungsorientierte Einführung. Berlin: Springer.

Best, Henning und Christof Wolf. 2010. Logistische Regression. S. 828-854 in Handbuch der sozialwissenschaftlichen Datenanalyse, hrsg. von Christof Wolf und Henning Best. Wiesbaden: VS Verlag für Sozialwissenschaften.

Bonß, Wolfgang und Sven Kesselring. 2002. Mobilität am Übergang von der Ersten zur Zweiten Moderne. S. 177-190 in Die Modernisierung der Moderne, hrsg. von Ulrich Beck und Wolfgang Bonß. Frankfurt a. M: Suhrkamp. 
Bühlmann, Marc. 2006. Politische Partizipation im kommunalen Kontext: Der Einfluss lokaler Kontexteigenschaften auf individuelles politisches Partizipationsverhalten. Bern: Haupt Verlag.

Bundesamt für Raumentwicklung. 2009. Faktenblatt. Zweitwohnungen der Schweizer Bevölkerung. Zusatzauswertung des Mikrozensus zum Verkehrsverhalten 2005. Bern: Eidgenössisches Departement für Umwelt, Verkehr, Energie und Kommunikation.

Case, Duncan. 1996. Contributions of journeys away to the definition of home: An empirical study of a dialectical process. Journal of Environmental Psychology 16(1): 1-15.

Dalton, Russell J. 2004. Democratic Challenges, Democratic Choices: The Erosion of Political Support in Advanced Political Democracies. Oxford: Oxford University Press.

Dalton, Russell J. 2008. The Good Citizen. How a Younger Generation is Reshaping American Politics. Washington D.C.: QC Press.

De Weerd, Marga und Bert Klandermans. 1999. Group identification and political protest: Farmer's protest in the Netherlands. European Journal of Social Psychology 29(8): 1073-1095.

Dittrich-Wesbuer, Andrea und Stefanie Föbker. 2013. Multilokales Wohnen - Verbreitung und Formen in Deutschland. S. 391-402 in Mobilitäten und Immobilitäten. Menschen - Ideen - Dinge - Kulturen - Kapital, hrsg. von Joachim Scheiner, Hans-Heinrich Blotevogel, Susanne Frank, Christian Holz-Rau und Nina Schuster. Essen: Klartext Verlag.

Dowding, Keith, Peter John, Thanos Mergroupis und Mark Van Vugt. 2000. Exit, voice and loyalty: Analytic and empirical developments. European Journal of Political Research 37(4): 469-495.

Finkel, Steven E. und Edward N. Muller. 1998. Rational choice and the dynamics of collective political action. Evaluating alternative models with panel data. American Political Science Review 92(1): 37-49.

Gabriel, Oscar W. 2013. Politische Partizipation. S. 381-411 in Politik im Kontext: Ist alle Politik lokale Politik?, hrsg. von Jan W. Van Deth und Markus Tausendpfund. Wiesbaden: Springer VS.

Gabriel, Oscar W. und Kerstin Völkl. 2005. Politische und soziale Partizipation. S. 523-573 in Handbuch Politisches System der Bundesrepublik Deutschland, hrsg. von Oscar W. Gabriel und Everhard Holtmann. München und Wien: Oldenbourg.

Hesse, Markus und Joachim Scheiner. 2007. Räumliche Mobilität im Kontext des sozialen Wandels: Eine Typologie multilokalen Wohnens. Geographische Zeitschrift 95(3): 138-154.

Hilti, Nicola. 2013. Lebenswelten multilokal Wohnender. Eine Betrachtung des Spannungsfeldes von Bewegung und Verankerung. Wiesbaden: Springer VS.

Hirschman, Albert O. 1970. Exit, Voice, and Loyalty. Responses to Decline in Firms, Organizations and States. Cambridge: Harvard University Press.

Jorgensen, Bradley und S. Richard C. Stedman. 2001. Sense of place as an attitude: Lakeshore owners' attitudes toward their properties. Journal of Environmental Psychology 21(3): 233-248.

Keil, Silke I. 2012. Partizipation und Ungleichheit. S. 173-208 in Deutschlands Metamorphosen. Ergebnisse des European Social Survey 2002 bis 2008, hrsg. von Silke I. Keil und Jan W. Van Deth. Baden-Baden: Nomos.

Koch, Andreas. 2008. Phänomene der Armut und Exklusion bei multilokalen Lebensformen. Mitteilungen der Österreichischen Geographischen Gesellschaft 150: 209-228.

Meil, Gerardo. 2008. Summary. Job mobility in Europe: Greater differences among social groups than among countries. S. 305-318 in Mobile Living Across Europe. Volume 1: Relevance and Diversity of Job-related Spatial Mobility in Six European Countries, hrsg. von Norbert F. Schneider und Gerardo Meil. Opladen: Barbara Budrich.

Menard, Scott. 2010. Logistic Regression. From Introductory to Advanced Concepts and Applications. London und Thousand Oaks: Sage Publications.

Mood, Carina. 2010. Logistic regression: Why we cannot do what we think we can do, and what we can do about it. European Sociological Review 26(1): 67-82. 
Mühler, Kurt und Karl-Dieter Opp. 2004. Region und Nation. Zu den Ursachen und Wirkungen regionaler und überregionaler Identifikation. Wiesbaden: VS Verlag für Sozialwissenschaften.

Mühler, Kurt und Karl-Dieter Opp. 2006. Region Nation Europa. Die Dynamik regionaler und überregionaler Identifikation. Wiesbaden: VS Verlag für Sozialwissenschaften.

Muller, Edward N. 1982. An explanatory model of differing types of participation. European Journal of Political Research 10(1): 1-16.

Nadler, Robert, Knut Petzold und Robert Schönduwe. 2015. Doing Online Surveys: Zum Einsatz in der sozialwissenschaftlichen Raumforschung. Raumforschung und Raumordnung 73(3): 203-217.

Norris, Pippa. 2002. Democratic Phoenix. Reinventing Political Acitivism. Cambridge: Cambridge University Press.

Olson, Mancur. 1965. The Logic of Collective Action. Cambridge: Schocken Books.

Opp, Karl-Dieter. 1986. Soft incentives and collective action. Participation in the anti-nuclear movement. British Journal of Political Science 16(1): 87-112.

Opp, Karl-Dieter. 1992. Legaler und illegaler Protest im interkulturellen Vergleich. Kölner Zeitschrift für Soziologie und Sozialpsychologie 44: 436-460.

Opp, Karl-Dieter und Steven E. Finkel. 2001. Politischer Protest, Rationalität und Lebensstile. Eine empirische Überprüfung alternativer Erklärungsmodelle. S. 73-108 in Politische Partizipation in der Bundesrepublik Deutschland. Empirische Befunde und theoretische Erklärungen, hrsg. von Achim Koch, Martina Wamser und Peter Schmidt. Opladen: Leske + Budrich.

Pampel, Fred C. 2000. Logistic Regression. A Primer. London und Thousand Oaks: Sage Publications.

Parry, Geraint, George Moyser und Neil Day. 1992. Political Participation and Democracy in Britain. Cambridge: Cambridge University Press.

Pattie, Charles, Patrick Seyd und Paul Whiteley. 2004. Citizenship in Britain. Values, Participation and Democracy. Cambridge: Cambridge University Press.

Petzold, Knut. 2011. Measuring "job-related" multi-locality - Overview and conceptual framework. S. 235-246 in Measuring Geographical Mobility in Regional Labour Market Monitoring, hrsg. von Christa Larsen, Ruth Hasberg, Alfons Schmid, Marc Bittner und Franz Clément. München: Rainer Hampp Verlag.

Petzold, Knut. 2013a. Multilokalität als Handlungssituation. Lokale Identifikation, Kosmopolitismus und ortsbezogenes Handeln unter Mobilitätsbedingungen. Wiesbaden: Springer VS.

Petzold, Knut. 2013b. Von einem, der auszog Wurzeln zu schlagen. Multilokalisierte Akteure und die Mechanismen lokaler Identifikation am Beispiel von Fernpendlern. Soziale Welt 64(3): 291-316.

Polletta, Francesta und James M. Jasper. 2001. Collective identity and social movements. Annual Review of Sociology 27(1): 283-305.

Putnam, Robert D. 1995. Bowling alone: America’s declining social capital. Journal of Democracy 6(1): 65-78.

Putnam, Robert D. 2000. Bowling Alone. The Collapse and Revival of American Community. New York: Simon \& Schuster.

Reuschke, Darja. 2010. Sozialer Wandel und multilokales Wohnen: Raum-zeitliche Muster multilokaler Wohnarrangements von Shuttles und Personen in einer Fernbeziehung. Wiesbaden: VS Verlag für Sozialwissenschaften.

Rüger, Heiko, Michaela Schier, Michael Feldhaus und Tammy Ries. 2014. Einstellungen zur Akzeptanz räumlicher Distanz in erwerbsbedingt multilokalen Lebensformen. Zeitschrift für Familienforschung 26(2): 121-143.

Schad, Helmut, Nicola Hilti, Margit Hugentobler und Cédric Duchêne-Lacroix. 2015. Multilokales Wohnen in der Schweiz - Erste Einschätzung zum Aufkommen und zu den Ausprägungen. S. 176-201 in Mobil und doppelt sesshaft. Studien zur residenziellen Multilokalität, hrsg. von Peter Weichhart und Peter Alexander Rumpolt. Wien: IfGR. 
Schneider, Norbert F., Ruth Limmer und Kerstin Ruckdeschel. 2001. Berufsmobilität und Lebensform Sind berufliche Mobilitätserfordernisse in Zeiten der Globalisierung noch mit Familie vereinbar? Stuttgart: Kohlhammer.

Sennett, Richard. 1998. Der flexible Mensch. Die Kultur des neuen Kapitalismus. Berlin: Berlin Verlag.

Sheller, Mimi und John Urry. 2006. The new mobilities paradigm. Environment and Planning A 38(2): 207-226.

Steinbrecher, Markus. 2009. Politische Partizipation in Deutschland. Baden-Baden: Nomos.

Sturm, Gabriele und Katrin Meyer. 2009. Was können Melderegister deutscher Großstädte zur Analyse residentieller Multilokalität beitragen? Informationen zur Raumentwicklung (IZR) 2009(1/2): 15-30.

Van Deth, Jan W. 2004. Soziale Partizipation. S. 295-315 in Deutschland in Europa. Ergebnisse des European Social Survey 2002-2003, hrsg. von Jan W. Van Deth. Wiesbaden: VS Verlag für Sozialwissenschaften.

Van der Land, Marco. 1998. Myths of mobility: On the geography of work and leisure among Rotterdam's new middle class. Journal of Housing and the Built Environment 13(2): 117-135.

Verba, Sidney, Kay Lehman Schlozman und Henry Brady. 1995. Voice and Equality. Civic Volontarism in American Politics. Cambridge: Harvard University Press.

Weichhart, Peter. 2009. Multilokalität - Konzepte, Theoriebezüge und Forschungsfragen. Informationen zur Raumentwicklung (IZR) 2009(1/2): 1-14.

Weiske, Christine, Knut Petzold und Diana Zierold. 2008. Multilokale Haushalte - mobile Gemeinschaften. Entwurf einer Typologie multilokaler Lebensformen. Sozialer Sinn. Zeitschrift für hermeneutische Sozialforschung 9(2): 281-300.

Weiske, Christine, Knut Petzold und Helmut Schad. 2015. Multilocal living - The approaches of rational choice theory, sociology of everyday life and actor-network theory. Journal of Economic and Social Geography (TESG) 106(4): 392-408. 


\section{$7 \quad$ Anhang}

\section{Tabelle A1 Angaben zu den Variablen}

\begin{tabular}{ll}
\hline Variablenname & $\begin{array}{l}\text { Bedeutung, Item, Frage. Wertebereich / Mittelwert / Standardabweichung } \\
\text { (ggf. nach Arbeits- und Primärort) }\end{array}$ \\
\hline $\begin{array}{l}\text { Freunde \& Verwandte am } \\
\text { Arbeitsort }\end{array}$ & $\begin{array}{l}\text { Wie viele Verwandte leben auch am Ort? Wie viele Personen Ihres Freundeskreises wohnen } \\
\text { auch am Ort? (0) ich habe keinen Freundeskreis/keine Verwandten; (1) weniger als die Häf- } \\
\text { te; (2) teils/teils; (3) mehr als die Hälfte. Aus beiden Indikatoren wurde ein additiver Index } \\
\text { gebildet. } \\
\text { Shuttles: 1-5 / 2.28 / 0.82. Locals: 1-5 / 3.44 / 1.04. }\end{array}$
\end{tabular}

Handlungsexklusivität am Arbeitsort

Identifikation mit dem Arbeitsort

Interesse am Arbeitsort

Lokale Wohndauer am Arbeitsort

Befristung der Multilokalität

Unzufriedenheit mit der lokalen Wohnsituation

Wahrgenommene lokale Verhaltenskontrolle

Politisches Engagement (Abhängige Variablen)
«Dieser Ort bietet mir Dinge, die mir sonst kein Ort bieten kann.»; «Dieser Ort ist der beste Ort, um Dinge zu tun die ich mag.»; «stimme nicht zu» (1) bis «stimme voll und ganz zu» (5). Aus beiden Indikatoren wurde eine additive Skala gebildet (Cronbachs $\alpha$ Arbeitsort $=0.749$; Primärort $=0.704$ ).

Shuttles: 1-5 / 2.50 / 1.02. Locals: 1-5/3.17 / 1.07.

«Wie stark fühlen Sie sich als Teil der örtlichen Bevölkerung?»; «Wie stark fühlen Sie sich mit dem Ort insgesamt verbunden?» ). (1) überhaupt nicht; (2) schwach; (3) teils/teils; (4) stark; (5) sehr stark.

Aus beiden Indikatoren wurde eine additive Skala gebildet gebildet (Cronbachs $\alpha$ Arbeitsort $=0.888$; Primärort $=0.857$ ).

Shuttles: 1-5 / 2.33 / 0.91. Locals: 1-5 / 3.54 / 1.00.

«Ich interessiere mich für das, was an diesem Ort geschieht.»; "stimme nicht zu» (1) bis "stimme voll und ganz zu» (5).

Shuttles: 1-5 / $3.41 / 1.11$. Locals: 1-5 / 3.96/1.33.

Wohndauer am Ort in Jahreskategorien zwischen (1) bis ein Jahr und (31) mehr als 30 Jahre. Shuttles: 1-31 / 3.66 / 4.92. Locals: 1-31/11.35/ 10.66.

Wie wahrscheinlich ist es, dass Sie zukünftig Ihr Leben an mehreren Orten zugunsten des Lebens an nur einem Ort aufgeben? «keinesfalls/wahrscheinlich nicht» (0); «ziemlich wahrscheinlich / ganz sicher» (1)

Alles in Allem: Wie zufrieden sind Sie mit Ihrer Wohnsituation am Ort insgesamt? (1) sehr zufrieden; (2) zufrieden; (3) weder zufrieden noch unzufrieden; (4) unzufrieden; (5) sehr unzufrieden.

Shuttles: 1-5 / 2.65 / 0.84. Locals: 1-5 / 2.02 / 0.82.

«Ich glaube, ich könnte die Gestaltung des Ortes beeinflussen.»; «Leute wie ich haben sowieso keinen Einfluss darauf, was hier vor Ort passiert.» (recodiert); «stimme nicht zu» (1) bis «stimme voll und ganz zu» (5). Aus beiden Indikatoren wurde eine additive Skala gebildet. (Cronbachs $\alpha$ Arbeitsort $=0.653$; Primärort $=0.590$ ).

Shuttles: 1-5 / 2.74 / 0.94. Locals: 1-5 / 3.27 / 0.97.

Haben Sie, seitdem Sie an diesem Ort wohnen, eine der folgenden Handlungen ausgeführt? (1) das kam für mich nicht infrage, (2) habe ich überlegt, aber nicht gemacht, (3) habe ich einmal gemacht, (4) habe ich mehrmals gemacht.

«Eintritt in eine an diesem Ort lokalpolitisch aktive Partei»

Shuttles: Arbeitsort: 1-4 / 1.13 / 0.47. Primärort: 1-4 / 1.42 / 0.75. Locals: 1-4 / 1.51 / 0.84. «Organisation von oder Teilnahme an Bürgerinitiativen»

Shuttles: Arbeitsort: 1-4 / 1.17 / 0.49. Primärort: 1-4 / 1.39 / 0.71. Locals: 1-4 / 1.50 / 0.86. «Spenden, z. B. an lokale Kirchen, Museen, Zoologische Gärten usw.»

Shuttles: Arbeitsort: 1-4 / 1.40 / 0.83. Primärort: 1-4 / 2.06 / 1.21. Locals: 1-4 / 2.23 / 1.18. «Organisation von oder Teilnahme an politischen Veranstaltungen/Demonstrationen» Shuttles: Arbeitsort: 1-4 / 1.74 / 1.08. Primärort: 1-4 / 2.63 / 1.32. Locals: 1-4 / 2.66 / 1.26. 


\section{Teilhabe}

vêrschįedenheitt

Dagmar Domenig,

und Urs Schäfer (Hrsg.)

Mediale Welt

inklusive!

Sichtbarkeit und

Teilhabe von

Menschen mit

Behinderungen

in den Medien

Dagmar Domenig und Urs Schäfer (Hrsg.)

Mediale Welt inklusive!

Sichtbarkeit und Teilhabe

von Menschen mit

Behinderungen in den

Medien

\section{Reihe}

«Teilhabe und Verschiedenheit»

184 Seiten, ISBN 978-3-03777-165-5, SFr. 29.-/Euro 25.-

Die Beiträge in diesem Buch beleuchten die Sichtbarkeit, die Repräsentation und die Partizipation von Menschen mit Behinderungen in den Medien. Dabei liegt der Fokus einerseits auf der Darstellung von Behinderung in der Öffentlichkeit und in den Massenmedien ganz allgemein, andererseits auf den typischen kommunikativen und gestalterischen Ausprägungen in der Fotografie und im Film sowie in der Spendenwerbung von Nonprofit-Organisationen. Ein weiteres Thema dieses Buches ist der barrierefreie Zugang zum Internet für Menschen mit einer kognitiven Beeinträchtigung. Die zentrale Forderung der Beiträge ist, den Menschen mit Behinderungen ihren Platz in der medialen Welt zuzugestehen, ohne sie damit zusätzlich zu stigmatisieren, zu erniedrigen, zu verniedlichen, zu verfremden oder zu romantisieren.
Die ganzseitigen Fotos stammen vom Fotografen Peter Dammann, der auch einen der Beiträge verfasst hat. Peter Dammann ist noch vor Fertigstellung des Buches verstorben.

Dr. Dagmar Domenig ist Juristin, Sozialanthropologin und Pflegefachfrau. Ihre Schwerpunktthemen sind Migration und Gesundheit, transkulturelle Kompetenz, Umgang mit Verschiedenheit im Gesundheits- und Sozialwesen. Seit 2011 ist Domenig Direktorin der Stiftung Arkadis in Olten (www.arkadis.ch).

Urs Schäfer ist seit 25 Jahren im NonprofitMarketing tätig. Die ersten 10 Jahre auf Agenturseite als Kommunikationsplaner und Berater, die folgenden Jahre als Fundraiser, Marketing- und Kommunikationsleiter bei diversen Nonprofit-Organisationen; aktuell bei der Stiftung Arkadis in Olten. 